\title{
A DUALITY FORMALISM IN THE SPIRIT OF GROTHENDIECK AND VERDIER
}

\author{
MITYA BOYARCHENKO AND VLADIMIR DRINFELD
}

\begin{abstract}
We study monoidal categories that enjoy a certain weakening of the rigidity property, namely, the existence of a dualizing object in the sense of Grothendieck and Verdier. We call them Grothendieck-Verdier categories. (They have also been studied in the literature under the name "*-autonomous categories.") Notable examples include the derived category of constructible sheaves on a scheme (with respect to tensor product) as well as the derived and equivariant derived categories of constructible sheaves on an algebraic group (with respect to convolution).

We show that the notions of pivotal category and ribbon category, which are well known in the setting of rigid monoidal categories, as well as certain standard results associated with these notions, have natural analogues in the world of Grothendieck-Verdier categories.
\end{abstract}

\section{Contents}

Introduction

Part 1. Formulations and easy proofs $\quad 6$

1. First properties of Grothendieck-Verdier categories 6

2. More examples of Grothendieck-Verdier categories $\quad 8$

3. Rigidity in r-categories $\quad 9$

4. $D^{2}$ as a monoidal equivalence 11

5. Pivotal structures on Grothendieck-Verdier categories 12

6. Braided Grothendieck-Verdier categories 14

7. Pivotal structures on braided Grothendieck-Verdier categories 17

8. Ribbon Grothendieck-Verdier categories 19

9. Relation between r-categories and Grothendieck-Verdier categories 20

2010 Mathematics Subject Classification. 18D10.

Key words and phrases. Monoidal category, braided category, pivotal structure, ribbon category, *autonomous category, Grothendieck-Verdier category.

M.B. (corresponding author) was supported by the NSF grant DMS-1001769.

V.D. was supported by the NSF grant DMS-1001660.

Addresses: M.B.: Department of Mathematics, University of Michigan, Ann Arbor, MI 48109.

V.D.: Department of Mathematics, University of Chicago, Chicago, IL 60637.

E-mail: mitya@math.uchicago.edu (M.B.), drinfeld@math.uchicago.edu (V.D.). 
Part 2. Proofs of the main results 21

10. Rigidity 21

11. The monoidal structure on $D^{2} \quad 23$

12. Proof of Lemma $6.8 \quad 25$

13. Proof of Proposition 5.7 26

14. Proof of Proposition 6.10 28

15. From Grothendieck-Verdier categories to r-categories 30

References $\quad 34$

\section{INTRODUCTION}

\subsection{Main definitions.}

Definition 0.1 . An object $K$ in a monoidal category $\mathcal{M}$ is said to be dualizing if for every $Y \in \mathcal{M}$ the functor $X \mapsto \operatorname{Hom}(X \otimes Y, K)$ is representable by some object $D Y \in \mathcal{M}$ and the contravariant functor $D: \mathcal{M} \longrightarrow \mathcal{M}$ is an antiequivalence. $D$ is called the duality functor with respect to $K$.

Remark 0.2. By Proposition 1.3 below, if a dualizing object exists then it is unique up to tensoring by an invertible object.

Definition 0.3. A Grothendieck-Verdier category is a pair $(\mathcal{M}, K)$, where $\mathcal{M}$ is a monoidal category and $K \in \mathcal{M}$ is a dualizing object.

Some examples of Grothendieck-Verdier categories are given in $\S 0.2$ below.

Remarks 0.4. (1) If $(\mathcal{M}, K)$ is a Grothendieck-Verdier category then $D: \mathcal{M} \longrightarrow \mathcal{M}$ will always denote the corresponding duality functor.

(2) By an abuse of language we will sometimes say "Grothendieck-Verdier category $\mathcal{M}$ " instead of "Grothendieck-Verdier category $(\mathcal{M}, K)$ ".

Definition 0.5. A monoidal category $\mathcal{M}$ is said to be an r-category if the unit object $\mathbb{1} \in \mathcal{M}$ is dualizing.

So any r-category can be considered as a Grothendieck-Verdier category with $K=\mathbb{1}$. The letter " $r$ ' in the name "r-category" is related to the words "rigid" and "regular", see Examples 0.8-0.9 below.

0.2. Main examples. Below we give some examples of Grothendieck-Verdier categories. More examples of such categories can be found in $\S 2$ and in the works by M. Barr, who studied them under the name of *-autonomous categories (e.g., see [Ba79, Ba95, Ba96, Ba99]).

Example 0.6. Let $\mathcal{M}=(\mathscr{D}(X), \otimes)$, where $X$ is a scheme of finite type over a field $k$ and $\mathscr{D}(X)=D_{c}^{b}\left(X, \overline{\mathbb{Q}}_{\ell}\right)$ is the bounded derived category of constructible $\ell$-adic sheaves on $X$, 
defined as in [Jan88, Ek90]. Let $K_{X} \in \mathscr{D}(X)$ be the dualizing complex. Then $\left(\mathcal{M}, K_{X}\right)$ is a Grothendieck-Verdier category. In this case $D$ is the usual Verdier duality functor $\mathbb{D}_{X}$.

Example 0.7. In [KS94] M. Kashiwara and P. Schapira introduce three variants of the category $\mathcal{M}$ from Example 0.6 in which sheaves are considered with respect to a usual topology (rather than a Grothendieck topology). More precisely, let $X$ be either a locally finite simplicial complex or a real-analytic manifold, or a complex-analytic one. In each of these situations they introduce in [KS94, ch. 8] a notion of constructibility for sheaves of abelian groups on $X$ so that the bounded derived category of constructible sheaves becomes a Grothendieck-Verdier category.

Example 0.8. Any rigid monoidal category ${ }^{1}$ is an r-category. The next example (or the elementary Example 2.3) shows that the converse is false.

Example 0.9. Let $X$ be a smooth scheme of pure dimension $d$ over a field $k$. Then the monoidal category $(\mathscr{D}(X), \otimes)$ is an r-category and $D: \mathscr{D}(X) \rightarrow \mathscr{D}(X)$ is the functor $N \longmapsto\left(\mathbb{D}_{X} N\right)[-2 d](-d)$. If $d>0$, then $(\mathscr{D}(X), \otimes)$ is not rigid because $D\left(M_{1} \otimes M_{2}\right) \neq$ $D\left(M_{2}\right) \otimes D\left(M_{1}\right)$ for some $M_{1}, M_{2} \in \mathscr{D}(X)$. For instance, let $M_{1}=M_{2}=i_{*} \overline{\mathbb{Q}}_{\ell}$, where $i:$ Spec $k \hookrightarrow X$ is a point. Then $D\left(M_{1} \otimes M_{2}\right)=D\left(i_{*} \overline{\mathbb{Q}}_{\ell}\right)=i_{*} \overline{\mathbb{Q}}_{\ell}[-2 d](-d)$, while $D\left(M_{2}\right) \otimes D\left(M_{1}\right)=i_{*} \overline{\mathbb{Q}}_{\ell}[-4 d](-2 d)$.

Example 0.10. Let $G$ be a group scheme of finite type over a field $k$. We define the equivariant derived category of $G$ as $\mathscr{D}_{G}(G):=D_{c}^{b}\left((\operatorname{Ad} G) \backslash G, \overline{\mathbb{Q}}_{\ell}\right)$ (i.e., $\mathscr{D}_{G}(G)$ is the bounded derived category of the quotient stack for the conjugation action of $G$ on itself [LO06]). The monoidal categories $\mathscr{D}(G)$ and $\mathscr{D}_{G}(G)$ equipped with the functor of convolution with compact support are r-categories with $D$ being the functor $\mathbb{D}_{G}^{-}=\mathbb{D}_{G} \circ \iota^{*}=\iota^{*} \circ \mathbb{D}_{G}$, where $\mathbb{D}_{G}$ is the Verdier duality functor on $G$ and $\iota: G \longrightarrow G$ is given by $g \mapsto g^{-1}$. The proof is straightforward and easy, see [BD11, Lemma A.10]. The monoidal category $\mathscr{D}_{G}(G)$ has a canonical braided structure, see [BD11, Definition A.43].

0.3. Subject of this work. Our goal is to establish some general facts about Grothendieck-Verdier categories, which are well known in the case of symmetric GrothendieckVerdier categories or in the case of arbitrary rigid monoidal categories. The proofs are not always straightforward generalizations of existing ones.

For instance, if $\mathcal{M}$ is a rigid monoidal category then the well known monoidal structure on the functor $D^{2}: \mathcal{M} \longrightarrow \mathcal{M}$ is usually defined via the canonical isomorphism $D(X \otimes$ $Y) \stackrel{\simeq}{\longrightarrow} D Y \otimes D X$. In an arbitrary Grothendieck-Verdier category (or even an arbitrary r-category) $D(X \otimes Y)$ is, in general, not isomorphic to $D Y \otimes D X$ (see Example 0.9). Nevertheless, the functor $D^{2}$ has a canonical monoidal structure, see $\S 4$.

Here is another example. It is well known that the set of twists $^{2}$ on a rigid braided category $\mathcal{M}$ is equipped with a canonical involution: namely, if $\theta \in \mathrm{Aut} \operatorname{Id}_{\mathcal{M}}$ is a twist

\footnotetext{
${ }^{1}$ The definition of rigidity is recalled in $\S 10.1$, see Definition 10.1 .

${ }^{2}$ The notion of twist is recalled in $\S 6.1$, see Definition 6.2 and Remark 6.6(i).
} 


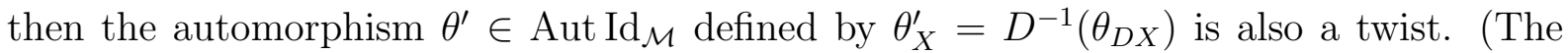
fixed points of this involution are called ribbon structures.) For arbitrary r-categories ${ }^{3}$ it is still true that $\theta^{\prime}$ is a twist (see Proposition 7.3 and Remark 7.4), but the proof has to be modified.

0.4. An $\infty$-categorical perspective (after J. Lurie). This subsection is informal. We hope that somebody will develop these ideas rigorously and systematically.

0.4.1. There is a general notion of $\mathcal{E}_{n}$-category, i.e., an $(\infty, 1)$-category ${ }^{4}$ with an action of the little $n$-disk operad $\mathcal{E}_{n}$. If $n=1$ and $n=2$ one gets, respectively, the notions of monoidal and braided $(\infty, 1)$-category.

An object of a monoidal $(\infty, 1)$-category is said to be dualizing if it is dualizing in its homotopy category (which is a usual monoidal category). Thus one has a notion of Grothendieck-Verdier $(\infty, 1)$-category. Since $\mathcal{E}_{1} \subset \mathcal{E}_{n}$ one has a notion of GrothendieckVerdier $\mathcal{E}_{n}$-category for each $n \geq 1$.

Example 0.11. The Grothendieck-Verdier categories from Example 0.6 and Examples 0.90.10 have natural $(\infty, 1)$-categorical "refinements". In particular, the "refinement" of the category $\mathscr{D}(G)$ from Example 0.10 is an $\mathcal{E}_{1}$-category and the "refinement" of $\mathscr{D}_{G}(G)$ is an $\mathcal{E}_{2}$-category.

0.4.2. As explained to us by J. Lurie, he expects (or at least, he does not exclude) that the results of $\S 4-8$ can be generalized to this setting and interpreted in terms of a certain canonical action of the topological group ${ }^{5} O(n+1)$ on the $\infty$-groupoid of GrothendieckVerdier $\mathcal{E}_{n}$-categories, whose restriction to $O(n) \subset O(n+1)$ comes from the obvious action of $O(n)$ on the operad $\mathcal{E}_{n}$. This would be very interesting. In Example 4.4.14 of [Lu09] Lurie sketches a construction of the $O(n+1)$-action on the space of rigid $\mathcal{E}_{n}$-categories.

Most of the results of our $\S \S 4-8$ (and their well known prototypes in the rigid case) can be interpreted from this perspective. For instance, the fact that any Grothendieck-Verdier category $\mathcal{M}$ has a canonical auto-equivalence (namely, $D^{2}: \mathcal{M} \stackrel{\sim}{\longrightarrow} \mathcal{M}$ ) is related to the canonical generator of $\pi_{1}(O(2))$, and the fact that for any braided Grothendieck-Verdier category $\mathcal{M}$ one has a canonical monoidal isomorphism $D^{4} \stackrel{\simeq}{\longrightarrow} \operatorname{Id}_{\mathcal{M}}$ (see $\S 6.3$ ) is related to the equality $\pi_{1}(O(3))=\mathbb{Z} / 2 \mathbb{Z}$.

0.4.3. A related idea is to regard an $\mathcal{E}_{n}$-category $\mathcal{M}$ as a fiber of a certain local system of $(\infty, 1)$-categories, $\mathfrak{M}$, over the sphere $S^{n}$. To define $\mathfrak{M}$, note that an $\mathcal{E}_{n}$-category $\mathcal{M}$ has not a single tensor product but rather a family of tensor products ${ }^{6} \otimes_{\omega}$ parameterized by $\omega \in S^{n-1}$. Accordingly, in a Grothendieck-Verdier $\mathcal{E}_{n}$-category $\mathcal{M}$ one has not a

\footnotetext{
${ }^{3}$ For Grothendieck-Verdier categories the statement has to be slightly modified, see Proposition 7.3.

${ }^{4} \mathrm{~A}(\infty, 1)$-category is an $\infty$-category in which all $m$-morphisms are invertible for $m>1$.

${ }^{5} \mathrm{An} \infty$-groupoid is essentially the same as a topological space, so it can be acted upon by a topological group.

${ }^{6}$ In the familiar case $n=1$ a monoidal category has two tensor products: the "original" one and the opposite one. In the Grothendieck-Verdier case the corresponding duality functors are $D$ and $D^{-1}$.
} 
single duality functor $D$ but rather a family of equivalences $D_{\omega}: \mathcal{M}^{\circ} \stackrel{\sim}{\longrightarrow} \mathcal{M}, \omega \in S^{n-1}$ (here $\mathcal{M}^{\circ}$ is the dual $(\infty, 1)$-category). To construct $\mathfrak{M}$, represent $S^{n}$ as the union of hemispheres $S_{ \pm}^{n}$, consider the constant sheaf on $S_{+}^{n}$ (resp. $S_{-}^{n}$ ) with fiber $\mathcal{M}$ (resp. $\mathcal{M}^{\circ}$ ) and glue them together using $D_{\omega}, \omega \in S^{n-1}=S_{+}^{n} \cap S_{-}^{n}$. Note that in general, $\mathfrak{M}$ is not a local system of $\mathcal{E}_{n}$-categories; in other words, the action of the loop space $\Omega S^{n}$ on $\mathcal{M}$ defined by $\mathfrak{M}$ does not preserve the $\mathcal{E}_{n}$-structure on $\mathcal{M}$. E.g., if $\mathcal{M}$ is a braided Grothendieck-Verdier category then the image in Aut $\left(\operatorname{Id}_{\mathcal{M}}\right)$ of the generator of $\pi_{1}\left(\Omega S^{2}\right)=\pi_{2}\left(S^{2}\right)$ equals the automorphism $C_{\mathcal{M}} \in \operatorname{Aut}\left(\operatorname{Id}_{\mathcal{M}}\right)$ from $\S 6.1$, which is a double-twist in the sense of Definition 6.5 and Remark 6.6(i) rather than a monoidal automorphism.

0.5. Structure of the article. We already defined the main objects of our study, Grothendieck-Verdier categories and r-categories, and remarked that every rigid monoidal category is an r-category. We begin the article by giving some basic properties of Grothendieck-Verdier categories in $§ 1$ and some further examples of (non-rigid) GrothendieckVerdier categories and r-categories in $\S 2$. In $\S 3$ we characterize rigid monoidal categories as r-categories satisfying a certain additional property.

We devote $§ \S 4-8$ to generalizations of certain well-known results and constructions involving rigid monoidal categories to the setting of Grothendieck-Verdier categories. In particular, in $\S 4$ we define a canonical monoidal structure on the square of the duality functor for an arbitrary Grothendieck-Verdier category. In $\$ 5$ we define and study pivotal structures on Grothendieck-Verdier categories. In $\S 6$ we study braided GrothendieckVerdier categories. In particular, we prove that for any such category the square of the duality functor is braided and its fourth power is canonically isomorphic to the identity functor. In $\S 7$ we analyze the relation between pivotal structures and twists on a braided Grothendieck-Verdier category. This leads us to introducing in $\S 8$ the notion of a ribbon Grothendieck-Verdier category (which specializes to the usual notion in the rigid case).

We end the first part of the article by answering in $\S 9$ the question of which Grothendieck-Verdier categories can be realized as Hecke subcategories of r-categories.

The second part of the article $(\S \S 10-15)$ is devoted to the proofs that are too long and/or too technical to be included into the first part (namely, the proofs of Propositions 1.5, 3.4, 4.2, 5.7, 6.10 and 9.4, as well as Lemma 6.8).

0.6. Acknowledgments. We thank J. Ayoub, A. Beilinson, P. Etingof, D. Gaitsgory, E. Jenkins, and especially J. Lurie for useful discussions and advice. We also thank the referee for helpful suggestions and for informing us about the articles [DS04] and [EMC10]. 


\section{Part 1. Formulations and easy proofs}

\section{First properties of Grothendieck-Verdier categories}

\subsection{Some canonical isomorphisms.}

Remarks 1.1. (i) By definition, in any Grothendieck-Verdier category $\mathcal{M}$ one has an isomorphism

$$
\operatorname{Hom}(X \otimes Y, K) \stackrel{\simeq}{\longrightarrow} \operatorname{Hom}(X, D Y)
$$

functorial in $X, Y \in \mathcal{M}$. Since $D$ is an antiequivalence the right-hand side of (1.1) identifies with $\operatorname{Hom}\left(Y, D^{-1} X\right)$. So one also has an isomorphism

$$
\operatorname{Hom}(X \otimes Y, K) \stackrel{\simeq}{\longrightarrow} \operatorname{Hom}\left(Y, D^{-1} X\right)
$$

functorial in $X, Y \in \mathcal{M}$. Thus a Grothendieck-Verdier category equipped with the opposite tensor product is still a Grothendieck-Verdier category, but D gets replaced by $D^{-1}$.

(ii) By (1.2), in any Grothendieck-Verdier category $\mathcal{M}$ one has a functorial isomorphism $\operatorname{Hom}\left(D^{2} Y \otimes X, K\right) \stackrel{\simeq}{\longrightarrow} \operatorname{Hom}(X, D Y)$. Combining it with (1.1) one gets a functorial isomorphism

$$
g: \operatorname{Hom}(X \otimes Y, K) \stackrel{\simeq}{\longrightarrow} \operatorname{Hom}\left(D^{2} Y \otimes X, K\right), \quad X, Y \in \mathcal{M} .
$$

Equivalently, $g$ is characterized by the commutativity of the diagram

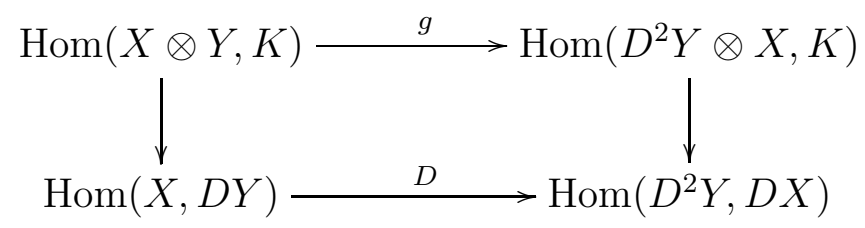

whose vertical arrows come from (1.1).

(iii) In any Grothendieck-Verdier category there exist right and left ${ }^{7}$ internal Hom's. More precisely, if one sets

$$
\underline{\operatorname{Hom}}(X, Z)=D^{-1}(D Z \otimes X)
$$

and

$$
\underline{\operatorname{Hom}}^{\prime}(Y, Z)=D\left(Y \otimes D^{-1} Z\right),
$$

then (1.1) and (1.2) yield functorial isomorphisms

$$
\operatorname{Hom}(X \otimes Y, Z) \stackrel{\simeq}{\longrightarrow} \operatorname{Hom}(Y, \underline{\operatorname{Hom}}(X, Z))
$$

\footnotetext{
${ }^{7}$ In this article we do not have to decide which of the two internal Hom's defined by (1.7) and (1.8) should be called "left." We prefer the convention that $\underline{\text { Hom }}$ is the right internal Hom and $\underline{H o m}^{\prime}$ is the left one. Reason: by Proposition 10.2, the right rigid dual of $X$ (if it exists) equals $\underline{\operatorname{Hom}}(\overline{X, \mathbb{1}})$. Note that the functor of left multiplication by $X$ is adjoint to the functor $\underline{\operatorname{Hom}}(X$, ?), which we would like to call the right internal Hom. We think this is acceptable.
} 
and

$$
\operatorname{Hom}(X \otimes Y, Z) \stackrel{\simeq}{\longrightarrow} \operatorname{Hom}\left(X, \underline{\operatorname{Hom}}^{\prime}(Y, Z)\right) .
$$

(iv) From (1.1) and (1.2) one gets canonical isomorphisms

$$
D \mathbb{1} \stackrel{\simeq}{\longrightarrow} K, \quad D^{-1} \mathbb{1} \stackrel{\simeq}{\longrightarrow} K .
$$

and therefore canonical isomorphisms

$$
\begin{aligned}
\mathbb{1} \stackrel{\simeq}{\longrightarrow} D^{2} \mathbb{1}, \\
K \stackrel{\simeq}{\longrightarrow} D^{2} K,
\end{aligned}
$$

where $(1.11)$ is the composition $K \stackrel{\simeq}{\longrightarrow} D \mathbb{1} \stackrel{\simeq}{\longrightarrow} D^{2} D^{-1} \mathbb{1} \stackrel{\simeq}{\longrightarrow} D^{2} K$.

(v) The inverse of (1.11) equals the image of $\operatorname{id}_{K} \in \operatorname{Hom}(\mathbb{1} \otimes K, K)$ under the isomorphism $\operatorname{Hom}(\mathbb{1} \otimes K, K) \stackrel{\simeq}{\longrightarrow} \operatorname{Hom}\left(D^{2} K \otimes \mathbb{1}, K\right)$ coming from (1.3). It is easy to check this using diagram (1.4) for $X=\mathbb{1}$ and $Y=K$.

1.2. Uniqueness of dualizing objects. Let us recall the following

Definition 1.2. If $\mathcal{M}$ is a monoidal category, an object $X \in \mathcal{M}$ is said to be invertible if there exists an object $Y \in \mathcal{M}$ such that $X \otimes Y \cong \mathbb{1} \cong Y \otimes X$.

Proposition 1.3. Let $(\mathcal{M}, K)$ be a Grothendieck-Verdier category.

(i) The functor $\mathscr{L} \mapsto D \mathscr{L}=K \otimes \mathscr{L}^{-1}$ is an antiequivalence between the full subcategory of invertible objects $\mathscr{L} \in \mathcal{M}$ and the full subcategory of dualizing objects.

(ii) The same is true for the functor $\mathscr{L} \mapsto D^{-1} \mathscr{L}=\mathscr{L}^{-1} \otimes K$.

(iii) If $\mathscr{L} \in \mathcal{M}$ is invertible then so is $D^{2} \mathscr{L}$ and one has a canonical isomorphism $K \otimes \mathscr{L}^{-1} \stackrel{\simeq}{\longrightarrow}\left(D^{2} \mathscr{L}\right)^{-1} \otimes K$.

Proof. By (1.6) and (1.8), an object $Z \in \mathcal{M}$ is dualizing if and only if the functor $Y \mapsto$ $Y \otimes D^{-1} Z$ is an equivalence. This means that $Z=D \mathscr{L}$, where $\mathscr{L}$ is invertible. In this case $D \mathscr{L}=K \otimes \mathscr{L}^{-1}$ by (1.1). We have proved (i). To prove (ii), use (1.5), (1.7), and (1.2) instead of (1.6), (1.8), and (1.1).

By (ii), $K \otimes \mathscr{L}^{-1}=D \mathscr{L}$ can also be written as $\widetilde{\mathscr{L}}^{-1} \otimes K=D^{-1} \widetilde{\mathscr{L}}$ for some invertible $\widetilde{\mathscr{L}} \in \mathcal{M}$. Since $D^{-1} \widetilde{\mathscr{L}}=D \mathscr{L}$ we have $\widetilde{\mathscr{L}}=D^{2} \mathscr{L}$.

Remark 1.4. Proposition 4.2 below yields a canonical isomorphism

$$
\left(D^{2} \mathscr{L}\right)^{-1} \stackrel{\simeq}{\longrightarrow} D^{2}\left(\mathscr{L}^{-1}\right) \text {. }
$$

1.3. Invertibility and rigidity of $K$. We learned the following statement from Dennis Gaitsgory. He also explained to us how it can be applied to studying the derived categories of $\mathcal{D}$-modules on certain algebraic stacks.

Proposition 1.5. A dualizing object of a monoidal category is invertible if and only if it is rigid in the sense of Definition 10.1.

See $§ 10.3$ for the proof. 


\section{More examples of GrothendiecK-Verdier CATEgories}

We already gave some examples in $§ 0.2$. More examples are below.

Example 2.1. As far as we understand, O. Gabber recently proved that $(\mathscr{D}(X), \otimes)$ is an r-category for any excellent regular scheme $X$ over $\mathbb{Z}\left[\ell^{-1}\right]$ (not necessarily of finite type).

Example 2.2. Here is a generalization of Example 0.10. Suppose we have a groupoid in the category of schemes of finite type over a field $k$. Let $\Gamma$ denote its "scheme of morphisms," and let $X$ denote its "scheme of objects," so one has the source and target maps $s, t: \Gamma \longrightarrow X$, the unit $1: X \longrightarrow \Gamma$, the inversion $\iota: \Gamma \stackrel{\simeq}{\longrightarrow} \Gamma$ and the product $\mu: \Gamma \times_{X} \Gamma \longrightarrow \Gamma$, where

$$
\Gamma \times_{X} \Gamma:=\left\{\left(\gamma_{1}, \gamma_{2}\right) \in \Gamma \times \Gamma \mid s\left(\gamma_{1}\right)=t\left(\gamma_{2}\right)\right\} .
$$

For $M_{1}, M_{2} \in \mathscr{D}(\Gamma)$ set $M_{1} * M_{2}:=\mu_{!}\left(p_{1}^{*} M_{1} \otimes p_{2}^{*} M_{2}\right)$, where $p_{1}, p_{2}: \Gamma \times_{X} \Gamma \longrightarrow \Gamma$ are the projections. Then $\mathscr{D}(\Gamma)$ becomes a monoidal category with unit object $\mathbb{1}=1$ ! $\overline{\mathbb{Q}}_{\ell}$. Define $K \in \mathscr{D}(\Gamma)$ by $K:=1_{*} K_{X}$, where $K_{X} \in \mathscr{D}(X)$ is the dualizing complex. Then $(\mathscr{D}(\Gamma), K)$ is a Grothendieck-Verdier category with duality functor $\mathbb{D}_{\Gamma}^{-}:=\mathbb{D}_{\Gamma} \circ \iota^{*}=\iota^{*} \circ \mathbb{D}_{\Gamma}$. Moreover, if an algebraic group $H$ acts on $(\Gamma, X, s, t, \mu)$ then $\left(\mathscr{D}_{H}(\Gamma), K\right)$ is a Grothendieck-Verdier category, where $\mathscr{D}_{H}(\Gamma):=D_{c}^{b}\left(H \backslash \Gamma, \overline{\mathbb{Q}}_{\ell}\right)$ is the bounded derived category [LO06] of the quotient stack $H \backslash \Gamma$. (The proof of these assertions is very similar to the proof of [BD11, Lemma A.10], so we omit it.) If $X$ is smooth and the embedding $1: X \longrightarrow \Gamma$ is closed then $1_{*} K_{X}=1_{!} K_{X}$ is an invertible object of $\mathscr{D}(\Gamma)$, so $\mathscr{D}(\Gamma)$ is an $r$-category. Note that if $\Gamma=X \times X$ then $1: X \longrightarrow \Gamma$ is the diagonal embedding, so the above closedness condition means that $X$ is separated.

The following elementary example of a non-rigid r-category is closely related to the works of Grothendieck in functional analysis. We learned this example from [Ba79].

Example 2.3. Let $\mathcal{M}$ be the category of finite-dimensional normed vector spaces over $\mathbb{R}$ with morphisms being linear operators of norm $\leq 1$. For $V, W \in \mathcal{M}$ define $V \otimes W$ to be the tensor product of vector spaces $V$ and $W$ equipped with the maximal norm such that $\|v \otimes w\| \leq\|v\| \otimes\|w\|$ for all $v \in V, w \in W$. The symmetric monoidal category $\mathcal{M}$ is an r-category with $D$ being the usual dual of a normed vector space. But $\mathcal{M}$ is not rigid. In fact, an object $V \in \mathcal{M}$ is rigid if and only if $\operatorname{dim} V \leq 1$ (to prove the "only if" statement, note that if $V$ is rigid then the composition of the coevaluation map $\mathbb{1} \longrightarrow V \otimes V^{*}$ and the evaluation map $V \otimes V^{*} \longrightarrow \mathbb{1}$ has norm $\leq 1$, where $V^{*}$ denotes the dual of $V$ ). By definition, $D V \otimes W$ identifies with the space $\operatorname{Hom}(V, W)$ equipped with the nuclear norm. On the other hand, one easily shows that $D(V \otimes D W)$ is the space $\operatorname{Hom}(V, W)$ equipped with the operator norm.

One can obtain more examples of Grothendieck-Verdier categories using Lemma 2.7 below. To formulate it, we need the following definition from [BD11, §2].

Definition 2.4. A morphism $\pi: \mathbb{1} \longrightarrow e$ in $\mathcal{M}$ is said to be an idempotent arrow if both morphisms $\pi \otimes \mathrm{id}_{e}: \mathbb{1} \otimes e \longrightarrow e \otimes e$ and $\mathrm{id}_{e} \otimes \pi: \mathbb{1} \otimes e \longrightarrow e \otimes e$ are isomorphisms. An 
object $e$ of a monoidal category $\mathcal{M}$ is said to be a closed idempotent ${ }^{8}$ if there exists an idempotent arrow $\mathbb{1} \longrightarrow e$.

Remark 2.5. In the situation of Example 0.10 with $G$ unipotent the categories $\mathscr{D}(G)$ and $\mathscr{D}_{G}(G)$ have many closed idempotents, see [BD11, $\S 1$ ] (especially $\S 1.11$ and Theorems 1.41(a), 1.49(c) from [BD11]).

If $e \in \mathcal{M}$ is a closed idempotent, we set

$$
e \mathcal{M} e:=\{X \in \mathcal{M} \mid X \cong e \otimes Y \otimes e \text { for some } Y \in \mathcal{M}\} .
$$

The tensor product of objects of $e \mathcal{M} e$ clearly belongs to $e \mathcal{M} e$. Equipped with this tensor product, $e \mathcal{M} e$ is a monoidal category with unit object $e$, see [BD11, Lemma 2.18]. More precisely, an idempotent arrow $\pi: \mathbb{1} \longrightarrow e$ defines a structure of unit object on $e$, see Lemma 9.1(b) below.

Definition 2.6. We call e $\mathcal{M} e \subset \mathcal{M}$ the Hecke subcategory of $\mathcal{M}$ defined by $e$.

Lemma 2.7. Let $(\mathcal{M}, K)$ be a Grothendieck-Verdier category, and let $e \in \mathcal{M}$ be a closed idempotent such that $D^{2} e \cong e$. Then De is a dualizing object of the monoidal category e $\mathcal{M} e$, so $(e \mathcal{M e}, D e)$ is a Grothendieck-Verdier category. In fact, $D(e \mathcal{M e})=e \mathcal{M e}$, and the duality functor for $(e \mathcal{M e}, D e)$ is isomorphic to the restriction of $D$ to e $\mathcal{M}$.

See [BD11, Lemma A.50] for a more precise version of Lemma 2.7 and a proof.

In $\S 9$ below we answer the following question: which Grothendieck-Verdier categories can be realized as Hecke subcategories of r-categories? For example, their class includes all additive Grothendieck-Verdier categories (apply Proposition 9.4 to $f=0$ ).

\section{RIGIDITY IN R-CATEGORIES}

3.1. The second tensor product in an r-category. Let $\mathcal{M}$ be an r-category. Define a new monoidal structure ${ }^{9} \odot: \mathcal{M} \times \mathcal{M} \rightarrow \mathcal{M}$ by

$$
X \odot Y:=D^{-1}(D Y \otimes D X) \text {. }
$$

Let us define a morphism

$$
X \otimes Y \rightarrow X \odot Y, \quad X, Y \in \mathcal{M} .
$$

To this end, note that by (1.1) we have canonical morphisms $D X \otimes X \rightarrow \mathbb{1}$ and $D Y \otimes Y \rightarrow$ $\mathbb{1}$. So we get a morphism $D Y \otimes D X \otimes X \otimes Y \rightarrow \mathbb{1}$, and by (1.2) this is the same as a morphism $X \otimes Y \rightarrow D^{-1}(D Y \otimes D X)=X \odot Y$. Clearly (3.2) is functorial in $X, Y$.

\footnotetext{
${ }^{8}$ In the situation of Definition 2.4 one has $e \otimes e \simeq e$, so the name "idempotent" is justified. The adjective "closed" is due to the fact that closed idempotents in the monoidal category $\mathcal{M}=\mathscr{D}(X)$ from Example 0.6 bijectively correspond to closed subsets $Y \subset X$. Namely, such $Y$ defines a closed idempotent $e=\left(\overline{\mathbb{Q}}_{\ell}\right)_{Y} \in \mathscr{D}(X)$, and the corresponding monoidal category (2.1) identifies with $\mathscr{D}(Y)$.

${ }^{9}$ One could also consider the monoidal structure given by $(X, Y) \longmapsto D\left(D^{-1} Y \otimes D^{-1} X\right)$, but Proposition 4.2 below allows us to identify it with $X \odot Y$.
} 
Lemma 3.1. The morphism (3.2) is compatible with the associativity constraints for $\otimes$ and $\odot$.

Proof. We have to show that the morphisms

$$
f: X_{1} \otimes X_{2} \otimes X_{3}=\left(X_{1} \otimes X_{2}\right) \otimes X_{3} \rightarrow\left(X_{1} \odot X_{2}\right) \odot X_{3}=X_{1} \odot X_{2} \odot X_{3}
$$

and

$$
g: X_{1} \otimes X_{2} \otimes X_{3}=X_{1} \otimes\left(X_{2} \otimes X_{3}\right) \rightarrow X_{1} \odot\left(X_{2} \odot X_{3}\right)=X_{1} \odot X_{2} \odot X_{3}
$$

coming from (3.2) are equal. By (1.1), we have canonical morphisms $D X_{i} \otimes X_{i} \rightarrow \mathbb{1}$ and therefore a morphism $h: D X_{3} \otimes D X_{2} \otimes D X_{1} \otimes X_{1} \otimes X_{2} \otimes X_{3} \rightarrow \mathbb{1}$. By (1.2), this is the same as a morphism $h^{\prime}: X_{1} \otimes X_{2} \otimes X_{3} \rightarrow X_{1} \odot X_{2} \odot X_{3}$. Both $f$ and $g$ equal $h^{\prime}$.

Remark 3.2. Since $\mathcal{M}$ is an r-category, $\mathbb{1}$ is a unit object for both $\otimes$ and $\odot$. It is not hard to check that the morphism (3.2) is compatible with the unit constraint for $\otimes$ and $\odot$.

Example 3.3. In the situation of Example 0.10 the monoidal functor (3.1) is the convolution without compact support and (3.2) is the usual morphism.

3.2. Rigidity in $r$-categories. Let us discuss the relation between the functor $D$ : $\mathcal{M} \longrightarrow \mathcal{M}$ and the notion of rigid duality from Definition 10.1.

Proposition 3.4. Let $\mathcal{M}$ be an r-category and $\epsilon: A \otimes B \longrightarrow \mathbb{1}$ a morphism in $\mathcal{M}$. Then $(B, \epsilon)$ is a right rigid dual of $A$ if and only if

(a) $\epsilon$ induces an isomorphism $B \stackrel{\simeq}{\longrightarrow} D^{-1} A$; and

(b) the canonical morphism $B \otimes A \longrightarrow B \odot A$ defined in $\S 3.1$ is an isomorphism.

In this case the canonical morphisms $B \otimes Y \longrightarrow B \odot Y$ and $Y \otimes A \longrightarrow Y \odot A$ are isomorphisms for all $Y \in \mathcal{M}$.

See $\S 10.2$ for the proof of the proposition.

Corollary 3.5. An object $X$ of an r-category $\mathcal{M}$ is rigid if and only if the canonical morphisms $X \otimes D X \longrightarrow X \odot D X$ and $D^{-1} X \otimes X \longrightarrow D^{-1} X \odot X$ defined in $\S 3.1$ are isomorphisms. Then the left rigid dual of $X$ equals $D X$, the right one equals $D^{-1} X$, and the canonical morphisms $X \otimes Y \longrightarrow X \odot Y$ and $Y \otimes X \longrightarrow Y \odot X$ are isomorphisms for all $Y \in \mathcal{M}$.

Corollary 3.6. The following properties of an r-category $\mathcal{M}$ are equivalent:

(i) $\mathcal{M}$ is rigid;

(ii) the canonical morphism $X \otimes Y \longrightarrow X \odot Y$ defined in $\S 3.1$ is an isomorphism for every $X, Y \in \mathcal{M}$;

(iii) the canonical morphism $X \otimes D X \longrightarrow X \odot D X$ is an isomorphism for every $X \in \mathcal{M}$.

Remark 3.7. Corollary 3.6 is probably well known; for instance, the equivalence $(i) \Longleftrightarrow$ (ii) is proved in the last paragraph of [DS04, Section 5]. 
Corollary 3.8. The monoidal category $\mathscr{D}(G)$ from Example 0.10 is rigid if and only if $G$ is proper. The same is true for $\mathscr{D}_{G}(G)$.

Proof. Let $\mathcal{M}$ be either $\mathscr{D}(G)$ or $\mathscr{D}_{G}(G)$. We use the equivalence (i) $\Leftrightarrow($ ii) from Corollary 3.6. Recall that $\otimes$ is convolution with compact support, $\odot$ is convolution without compact support, and the morphism $f_{X Y}: X \otimes Y \longrightarrow X \odot Y$ is the usual one (see Example 3.3). So if $G$ is proper then $f_{X Y}$ is an isomorphism for all $X, Y \in \mathcal{M}$. Conversely, if $f_{X Y}$ is an isomorphism for $X=Y=\overline{\mathbb{Q}}_{\ell}$ (the constant sheaf on $G$ ), then $G$ is proper.

\section{4. $D^{2}$ AS A MONOIDAL EQUIVALENCE}

By (1.3), for each $X, Y_{1}, Y_{2} \in \mathcal{M}$ one has a canonical isomorphism

$$
\operatorname{Hom}\left(X \otimes Y_{1} \otimes Y_{2}, K\right) \stackrel{\simeq}{\longrightarrow} \operatorname{Hom}\left(D^{2}\left(Y_{1} \otimes Y_{2}\right) \otimes X, K\right)
$$

On the other hand, writing $X \otimes Y_{1} \otimes Y_{2}$ as $\left(X \otimes Y_{1}\right) \otimes Y_{2}$ and applying (1.3) twice one gets an isomorphism

$$
\operatorname{Hom}\left(X \otimes Y_{1} \otimes Y_{2}, K\right) \stackrel{\simeq}{\longrightarrow} \operatorname{Hom}\left(D^{2} Y_{1} \otimes D^{2} Y_{2} \otimes X, K\right) .
$$

Combining (4.1) and (4.2) one gets a functorial isomorphism

$$
\operatorname{Hom}\left(D^{2}\left(Y_{1} \otimes Y_{2}\right) \otimes X, K\right) \stackrel{\simeq}{\longrightarrow} \operatorname{Hom}\left(D^{2} Y_{1} \otimes D^{2} Y_{2} \otimes X, K\right), \quad X, Y_{1}, Y_{2} \in \mathcal{M} .
$$

Lemma 4.1. Let $(\mathcal{M}, K)$ be a Grothendieck-Verdier category and $Z_{1}, Z_{2} \in \mathcal{M}$. Then every morphism $\operatorname{Hom}\left(Z_{1} \otimes X, K\right) \rightarrow \operatorname{Hom}\left(Z_{2} \otimes X, K\right)$ functorial in $X \in \mathcal{M}$ comes from a unique morphism $Z_{2} \longrightarrow Z_{1}$.

Proof. Use the isomorphism $\operatorname{Hom}\left(Z_{i} \otimes X, K\right) \stackrel{\simeq}{\longrightarrow} \operatorname{Hom}\left(Z_{i}, D X\right)$ and Yoneda's lemma.

Lemma 4.1 shows that the isomorphism (4.3) comes from a unique functorial isomorphism

$$
u_{Y_{1}, Y_{2}}: D^{2}\left(Y_{1} \otimes Y_{2}\right) \stackrel{\simeq}{\longrightarrow} D^{2} Y_{1} \otimes D^{2} Y_{2}, \quad Y_{1}, Y_{2} \in \mathcal{M}
$$

Proposition 4.2. The isomorphism (4.4) defines a monoidal structure on the functor $D^{2}: \mathcal{M} \stackrel{\sim}{\longrightarrow} \mathcal{M}$. The corresponding isomorphism $\mathbb{1} \stackrel{\simeq}{\longrightarrow} D^{2}(\mathbb{1})$ is equal to $(1.10)$.

As explained to us by J. Ayoub, this can be checked directly (by Lemma 4.1, to prove that the two isomorphisms $D^{2}\left(Y_{1} \otimes Y_{2} \otimes Y_{3}\right) \stackrel{\simeq}{\longrightarrow} D^{2} Y_{1} \otimes D^{2} Y_{2} \otimes D^{2} Y_{3}$ are equal, it suffices to show that the corresponding isomorphisms

$$
\operatorname{Hom}\left(D^{2}\left(Y_{1} \otimes Y_{2} \otimes Y_{3}\right) \otimes X, K\right) \stackrel{\simeq}{\longrightarrow} \operatorname{Hom}\left(D^{2} Y_{1} \otimes D^{2} Y_{2} \otimes D^{3} Y_{3} \otimes X, K\right)
$$

are equal). In $\S 11.1$ we give a slightly different proof of Proposition 4.2. 


\section{Pivotal structures on Grothendieck-Verdier Categories}

\subsection{The definition of pivotal structure.}

Definition 5.1. A pivotal structure on a Grothendieck-Verdier category $(\mathcal{M}, K)$ is a functorial isomorphism

$$
\psi_{X, Y}: \operatorname{Hom}(X \otimes Y, K) \stackrel{\simeq}{\longrightarrow} \operatorname{Hom}(Y \otimes X, K), \quad X, Y \in \mathcal{M}
$$

such that

$$
\begin{gathered}
\psi_{X \otimes Y, Z} \circ \psi_{Y \otimes Z, X} \circ \psi_{Z \otimes X, Y}=\mathrm{id}, \quad X, Y, Z \in \mathcal{M} ; \\
\psi_{X, Y} \circ \psi_{Y, X}=\mathrm{id}, \quad X, Y \in \mathcal{M} .
\end{gathered}
$$

In particular, one has a notion of pivotal structure on an r-category (which can be considered as a Grothendieck-Verdier category with $K=\mathbb{1}$ ).

Definition 5.2. A pivotal Grothendieck-Verdier category is a Grothendieck-Verdier category with a pivotal structure. A pivotal r-category is an r-category with a pivotal structure.

The name "pivotal category" goes back to [FY89, Definition 1.3].

Lemma 5.3. Let $\mathcal{M}$ be a Grothendieck-Verdier category and $\psi$ an isomorphism (5.1) satisfying (5.2). Then $\psi$ satisfies (5.3) if and only if $\psi_{K, \mathbb{1}}=\mathrm{id}$.

Proof. Setting $Z=\mathbb{1}$ in (5.2) we see that (5.3) holds if and only if the isomorphism $\psi_{X, \mathbb{1}}: \operatorname{Hom}(X, K) \rightarrow \operatorname{Hom}(X, K)$ equals the identity for all $X$. By Yoneda's lemma, this happens if and only if $\psi_{K, \mathbb{1}}=$ id.

Corollary 5.4. If $\mathcal{M}$ is an r-category then (5.2) implies (5.3).

Remark 5.5. By (5.2) and (5.3), a pivotal structure on a Grothendieck-Verdier category defines for any integers $n \geq m \geq 1$ a canonical isomorphism

$$
\operatorname{Hom}\left(X_{1} \otimes \ldots \otimes X_{n}, K\right) \stackrel{\simeq}{\longrightarrow} \operatorname{Hom}\left(X_{m} \otimes \ldots \otimes X_{n} \otimes X_{1} \otimes \ldots \otimes X_{m-1}, K\right), X_{i} \in \mathcal{M} .
$$

5.2. Pivotal structures and isomorphisms $\mathrm{Id} \stackrel{\simeq}{\longrightarrow} D^{2}$.

Lemma 5.6 (Cf. [EMC10]). There is a one-to-one correspondence between functorial isomorphisms

$$
\psi_{X, Y}: \operatorname{Hom}(X \otimes Y, K) \stackrel{\simeq}{\longrightarrow} \operatorname{Hom}(Y \otimes X, K), \quad X, Y \in \mathcal{M}
$$

and isomorphisms of functors $f: \operatorname{Id}_{\mathcal{M}} \stackrel{\simeq}{\longrightarrow} D^{2}$. Namely, $\psi$ corresponds to $f$ if the diagram

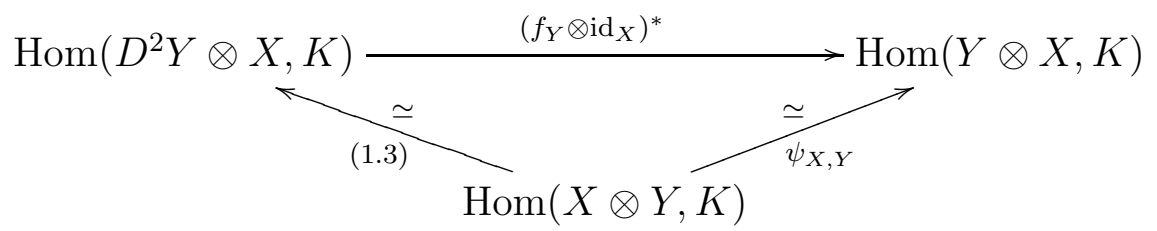

commutes for all $X, Y \in \mathcal{M}$. Here the left diagonal arrow is the isomorphism (1.3). 
Proof. Use Lemma 4.1.

Proposition 5.7. An isomorphism $f: \operatorname{Id}_{\mathcal{M}} \stackrel{\simeq}{\longrightarrow} D^{2}$ corresponds (in the sense of Lemma 5.6) to a pivotal structure if and only if it satisfies the following conditions:

(i) $f$ is monoidal; and

(ii) $f_{K}: K \stackrel{\simeq}{\longrightarrow} D^{2} K$ equals the isomorphism (1.11).

In this case

$$
f_{D X}=D\left(f_{X}\right)^{-1}, \quad \forall X \in \mathcal{M} .
$$

The proof is given in $\S 13$.

Remarks 5.8. (i) If $\mathcal{M}$ is an r-category then condition (ii) from Proposition 5.7 clearly follows from condition (i). For more general Grothendieck-Verdier categories this is not always the case. For instance, consider the pre-additive category $\mathcal{M}$ with objects $0, \mathbb{1}, K$ and with

$$
\operatorname{Hom}(\mathbb{1}, K)=\operatorname{Hom}(K, \mathbb{1})=0, \quad \text { End } \mathbb{1}=\text { End } K=A,
$$

where $A$ is a commutative unital ring. Define the tensor product $\mathcal{M} \otimes \mathcal{M} \rightarrow \mathcal{M}$ on objects so that $K \otimes K=0$ and $\mathbb{1} \otimes X=X \otimes \mathbb{1}=X$ for all $X \in \mathcal{M}$, define it on morphisms using the product in $A$, and take the associativity constraint in $\mathcal{M}$ to be trivial. Then $\mathcal{M}$ is a Grothendieck-Verdier category. In this situation monoidal isomorphisms Id $\stackrel{\simeq}{\longrightarrow} D^{2}$ bijectively correspond to elements of $A^{\times}$, and only one of them defines a pivotal structure (namely, the isomorphism corresponding to $1 \in A^{\times}$).

(ii) By the previous remark, in the case of r-categories a pivotal structure can equivalently be defined to be a monoidal isomorphism $f: \mathrm{Id} \stackrel{\simeq}{\longrightarrow} D^{2}$. It is this definition that was used in works on rigid monoidal categories (e.g., see [ENO05, Definition $2.7])$.

(iii) Here is a way to combine the two conditions on $f$ from Proposition 5.7 into one. Let $\mathfrak{A}$ be the 2-groupoid of pairs consisting of a monoidal category and an object in it. A Grothendieck-Verdier category $(\mathcal{M}, K)$ is an object in $\mathfrak{A}$. The monoidal structure on $D^{2}$ and the isomorphism $K \stackrel{\simeq}{\longrightarrow} D^{2}(K)$ defined in Remark 1.1(iv) allow us to consider $D^{2}$ as a 1-automorphism of $(\mathcal{M}, K) \in \mathfrak{A}$. The two conditions on $f$ from Proposition 5.7 mean that $f: \mathrm{Id} \stackrel{\simeq}{\longrightarrow} D^{2}$ is a 2-isomorphism in $\mathfrak{A}$.

\subsection{Examples of pivotal Grothendieck-Verdier categories.}

Example 5.9. Every symmetric Grothendieck-Verdier category has an obvious pivotal structure.

Example 5.10. The categories $\mathscr{D}(G)$ and $\mathscr{D}_{G}(G)$ from Example 0.10 have a canonical pivotal structure (see [BD11, §A.2.3]). The corresponding isomorphism Id $\stackrel{\simeq}{\longrightarrow} D^{2}=$ $\left(\mathbb{D}_{G} \circ \iota^{*}\right)^{2}$ comes from the obvious isomorphisms $\left(\mathbb{D}_{G}\right)^{2} \stackrel{\simeq}{\longrightarrow} \mathrm{Id},\left(\iota^{*}\right)^{2} \stackrel{\simeq}{\longrightarrow} \mathrm{Id}, \mathbb{D}_{G} \circ \iota^{*} \stackrel{\simeq}{\longrightarrow}$ $\iota^{*} \circ \mathbb{D}_{G}$. 
Example 5.11. Quite similarly to the previous example, one defines a canonical pivotal structure on the Grothendieck-Verdier category $\mathscr{D}(\Gamma)$ from Example 2.2.

\section{Braided GrothendieCK-VERdier CATEGories}

A braided Grothendieck-Verdier category is a Grothendieck-Verdier category $(\mathcal{M}, K)$ equipped with a braiding $\beta_{X, Y}: X \otimes Y \stackrel{\simeq}{\longrightarrow} Y \otimes X$. For any Grothendieck-Verdier category $(\mathcal{M}, K)$ the functor $D^{2}: \mathcal{M} \stackrel{\sim}{\longrightarrow} \mathcal{M}$ has a canonical monoidal structure, see $\S 4$. The main goal of this section is to prove the following proposition.

Proposition 6.1. Let $(\mathcal{M}, K, \beta)$ be a braided Grothendieck-Verdier category. Then

(i) the monoidal functor $D^{2}: \mathcal{M} \stackrel{\sim}{\longrightarrow} \mathcal{M}$ is braided;

(ii) there is a canonical monoidal isomorphism ${ }^{10} D^{4} \stackrel{\simeq}{\longrightarrow} \operatorname{Id}_{\mathcal{M}}$.

To prove Proposition 6.1, we will construct a monoidal equivalence between each of the monoidal functors $D^{ \pm 2}$ and a certain braided equivalence $J_{\mathcal{M}}: \mathcal{M} \stackrel{\sim}{\longrightarrow} \mathcal{M}$, which was defined by Joyal and Street for any braided category $\mathcal{M}{ }^{11}$ The definition of $J_{\mathcal{M}}$ is recalled in $\S 6.1$, and the canonical monoidal isomorphisms $\vartheta^{ \pm}: J_{\mathcal{M}}^{ \pm 1} \stackrel{\simeq}{\longrightarrow} D^{2}$ are constructed in $\S 6.2$. Using $\vartheta^{ \pm}$we define in $\S 6.3$ a canonical monoidal isomorphism $\gamma_{\mathcal{M}}: \operatorname{Id}_{\mathcal{M}} \stackrel{\simeq}{\longrightarrow} D^{4}$ and a certain monoidal isomorphism $C_{\mathcal{M}}: \operatorname{Id}_{\mathcal{M}} \stackrel{\simeq}{\longrightarrow} J_{\mathcal{M}}^{2}$, which we call the canonical double-twist. In fact, $J_{\mathcal{M}}$ is just the identity functor equipped with a nontrivial monoidal structure, so one can consider $C_{\mathcal{M}}$ as a (non-monoidal) automorphism of $\operatorname{Id}_{\mathcal{M}}{ }^{12}$

\subsection{The Joyal-Street equivalence, twists, and double-twists.}

Definition 6.2. Let $\mathcal{M}$ be a braided category. The Joyal-Street equivalence is the following braided equivalence $J=J_{\mathcal{M}}: \mathcal{M} \stackrel{\simeq}{\longrightarrow} \mathcal{M}$ : as a functor, $J_{\mathcal{M}}=\operatorname{Id}_{\mathcal{M}}$, but the isomorphism $J_{\mathcal{M}}(X \otimes Y) \stackrel{\simeq}{\longrightarrow} J_{\mathcal{M}}(X) \otimes J_{\mathcal{M}}(Y)$ equals

$$
\beta_{Y, X} \circ \beta_{X, Y}: X \otimes Y \stackrel{\simeq}{\longrightarrow} X \otimes Y \text {. }
$$

Lemma 6.3. The isomorphism (6.1) indeed defines a braided structure on the identity functor $\operatorname{Id}_{\mathcal{M}}: \mathcal{M} \longrightarrow \mathcal{M}$.

We learned this lemma and its proof given below from [JS93, Remark 6.1].

Proof. For $X, Y \in \mathcal{M}$ we write $\beta_{X, Y}^{+}=\beta_{X, Y}: X \otimes Y \stackrel{\simeq}{\longrightarrow} Y \otimes X$ and $\beta_{X, Y}^{-}=\beta_{Y, X}^{-1}$ : $X \otimes Y \stackrel{\simeq}{\longrightarrow} Y \otimes X$. Let $\mathcal{M}^{\text {opp }}$ be the monoidal category opposite to $\mathcal{M}$; thus $\mathcal{M}^{\text {opp }}$ equals $\mathcal{M}$ as a category, but the monoidal structure is given by $X \stackrel{o p p}{\otimes} Y=Y \otimes X$. Then $J_{\mathcal{M}}$ is equal to $\Phi^{+}\left(\Phi^{-}\right)^{-1}$, where $\Phi^{ \pm}: \mathcal{M}^{\text {opp }} \stackrel{\sim}{\longrightarrow} \mathcal{M}$ are the following monoidal equivalences:

\footnotetext{
${ }^{10}$ Recall that a braided isomorphism between braided functors is the same as a monoidal isomorphism.

${ }^{11}$ In terms of $\S 0.4, J_{\mathcal{M}}$ comes from the action of $S O(2)$ on the operad $\mathcal{E}_{2}$.

${ }^{12}$ This automorphism is the image of the generator of $\pi_{1}\left(\Omega S^{2}\right)=\pi_{2}\left(S^{2}\right)$ under the action of $\Omega S^{2}$ on $\mathcal{M}$ mentioned in $\S 0.4$. Note that since our $\mathcal{M}$ is a usual category rather than an $(\infty, 1)$-category this action does not feel $\pi_{i}\left(\Omega S^{2}\right)=\pi_{i+1}\left(S^{2}\right)$ for $i>1$.
} 
as a functor, $\Phi^{ \pm}$equals $\operatorname{Id}_{\mathcal{M}}$, and the isomorphism from $\Phi^{ \pm}(X) \otimes \Phi^{ \pm}(Y)=X \otimes Y$ to $\Phi^{ \pm}(X \otimes Y)=Y \otimes X$ equals $\beta_{X, Y}^{ \pm}$. (The fact that $\Phi^{ \pm}$are indeed monoidal functors follows immediately from the hexagon axioms.) Moreover, if we equip $\mathcal{M}^{\text {opp }}$ with the braiding

$$
X \stackrel{o p p}{\otimes} Y=Y \otimes X \stackrel{\beta_{Y, X}}{\longrightarrow} X \otimes Y=Y \stackrel{o p p}{\otimes} X
$$

then $\Phi^{ \pm}$become braided monoidal functors (here the verification is a tautology), which implies that $J_{\mathcal{M}}$ is also braided.

Remark 6.4. $J_{\mathcal{M}}^{-1}$ is the functor $\operatorname{Id}_{\mathcal{M}}: \mathcal{M} \longrightarrow \mathcal{M}$ equipped with the braided structure

$$
\left(\beta_{Y, X} \circ \beta_{X, Y}\right)^{-1}: X \otimes Y \stackrel{\simeq}{\longrightarrow} X \otimes Y .
$$

In other words, $J_{\mathcal{M}}^{-1}$ is the Joyal-Street equivalence for $\mathcal{M}$ equipped with the opposite braiding $\beta_{X, Y}^{-}:=\beta_{Y, X}^{-1}$.

Definition 6.5. A twist on a braided category $\mathcal{M}$ is a monoidal isomorphism $\theta: \operatorname{Id}_{\mathcal{M}} \stackrel{\simeq}{\longrightarrow}$ $J_{\mathcal{M}}$, where $J_{\mathcal{M}}$ is the Joyal-Street equivalence (see Definition 6.2). A double-twist on $\mathcal{M}$ is a monoidal isomorphism $\operatorname{Id}_{\mathcal{M}} \stackrel{\simeq}{\longrightarrow} J_{\mathcal{M}}^{2}$.

Remarks 6.6. (i) It is easy to check that the above definition of twist is equivalent to the usual one, i.e., a twist is an automorphism $\theta$ of the identity functor on $\mathcal{M}$ that satisfies

$$
\theta_{X \otimes Y}=\beta_{Y, X} \circ \beta_{X, Y} \circ\left(\theta_{X} \otimes \theta_{Y}\right) \quad \forall X, Y \in \mathcal{M} .
$$

Similarly, a double-twist is an automorphism $f$ of the identity functor such that

$$
f_{X \otimes Y}=\left(\beta_{Y, X} \circ \beta_{X, Y}\right)^{2} \circ\left(f_{X} \otimes f_{Y}\right) \quad \forall X, Y \in \mathcal{M} .
$$

(ii) The previous remark implies that for any twist $\theta$ one has $\theta_{\mathbb{1}}=\mathrm{id}_{\mathbb{1}}$ and for any double-twist $f$ one has $f_{\mathbb{1}}=\mathrm{id} \mathbb{d}_{\mathbb{1}}$.

(iii) If $\theta_{1}, \theta_{2}: \operatorname{Id}_{\mathcal{M}} \stackrel{\simeq}{\longrightarrow} J_{\mathcal{M}}$ are twists then $\theta_{1} \theta_{2}: \operatorname{Id}_{\mathcal{M}} \stackrel{\simeq}{\longrightarrow} J_{\mathcal{M}}^{2}$ is a double-twist. Moreover, $\theta_{1} \theta_{2}=\theta_{2} \theta_{1}$. Indeed, $J_{\mathcal{M}}$ equals $\operatorname{Id}_{\mathcal{M}}$ as a functor, so for each $X \in \mathcal{M}$ the isomorphism $\left(\theta_{i}\right)_{X}$ belongs to the center of Aut $X$ and $\left(\theta_{1} \theta_{2}\right)_{X}=\left(\theta_{1}\right)_{X} \circ\left(\theta_{2}\right)_{X}=$ $\left(\theta_{2}\right)_{X} \circ\left(\theta_{1}\right)_{X}=\left(\theta_{2} \theta_{1}\right)_{X}$

(iv) The set of all twists is either empty or a torsor over $\operatorname{Aut}^{\otimes}\left(\operatorname{Id}_{\mathcal{M}}\right)$, i.e., the group of monoidal automorphisms of $\mathrm{Id}_{\mathcal{M}}$. The same is true for double-twists. The map $\left(\theta_{1}, \theta_{2}\right) \mapsto \theta_{1} \theta_{2}$ from Remark (iii) agrees with the action of $\operatorname{Aut}^{\otimes}\left(\operatorname{Id}_{\mathcal{M}}\right)$.

6.2. The canonical monoidal isomorphisms $J_{\mathcal{M}} \stackrel{\simeq}{\longrightarrow} D^{2} \stackrel{\simeq}{\longleftarrow} J_{\mathcal{M}}^{-1}$. Let $(\mathcal{M}, K, \beta)$ be a braided Grothendieck-Verdier category. As in the proof of Lemma 6.3, we write $\beta_{X, Y}^{+}=\beta_{X, Y}$ and $\beta_{X, Y}^{-}=\beta_{Y, X}^{-1}$ for all $X, Y \in \mathcal{M}$.

Definition 6.7. For each $Y \in \mathcal{M}$, we let $\vartheta_{Y}^{ \pm}: Y \stackrel{\simeq}{\longrightarrow} D^{2} Y$ be the unique isomorphism ${ }^{13}$ such that for every $X \in \mathcal{M}$, the induced map

$$
\operatorname{Hom}\left(D^{2} Y \otimes X, K\right) \longrightarrow \operatorname{Hom}(Y \otimes X, K)
$$

\footnotetext{
${ }^{13}$ The existence and uniqueness of $\vartheta_{Y}^{ \pm}$follows from Lemma 4.1
} 
is equal to the composition

$$
\operatorname{Hom}\left(D^{2} Y \otimes X, K\right) \stackrel{\simeq}{\longrightarrow} \operatorname{Hom}(X \otimes Y, K) \stackrel{\left(\beta_{Y, X}^{ \pm}\right)^{*}}{\longrightarrow} \operatorname{Hom}(Y \otimes X, K),
$$

where the first arrow is inverse to the isomorphism (1.3).

Clearly $\vartheta_{Y}^{ \pm}$is functorial in $Y$, so we have isomorphisms of functors $\vartheta^{ \pm}: \operatorname{Id}_{\mathcal{M}} \stackrel{\simeq}{\longrightarrow} D^{2}$. The next lemma may be considered as an equivalent definition of $\vartheta^{ \pm}$.

Lemma 6.8. Let $(\mathcal{M}, K, \beta)$ be a braided Grothendieck-Verdier category, and let $\varphi^{ \pm}$: $D^{-1} \stackrel{\simeq}{\longrightarrow} D$ be the isomorphisms induced by the compositions

$$
\operatorname{Hom}\left(Y, D^{-1} X\right) \stackrel{\simeq}{\longrightarrow} \operatorname{Hom}(X \otimes Y, K) \stackrel{\left(\beta_{Y, X}^{ \pm}\right)^{*}}{\longrightarrow} \operatorname{Hom}(Y \otimes X, K) \stackrel{\simeq}{\longrightarrow} \operatorname{Hom}(Y, D X)
$$

for all $X, Y \in \mathcal{M}$. Then

$$
\vartheta_{Y}^{ \pm}=\varphi_{D Y}^{ \pm} \quad \text { for all } Y \in \mathcal{M}
$$

and

$$
D\left(\varphi_{X}^{ \pm}\right)=\left(\varphi_{D X}^{\mp}\right)^{-1} \quad \text { for all } X \in \mathcal{M} \text {. }
$$

The lemma will be proved in $\S 12.2$.

Remark 6.9. In view of Lemma 6.8, we have

$$
\vartheta_{D X}^{ \pm}=D\left(\vartheta_{X}^{\mp}\right)^{-1} \quad \text { for all } X \in \mathcal{M} \text {. }
$$

By Proposition 4.2, the functor $D^{2}: \mathcal{M} \stackrel{\simeq}{\longrightarrow} \mathcal{M}$ is equipped with a canonical monoidal structure. On the other hand, we have the Joyal-Street monoidal equivalence $J_{\mathcal{M}}: \mathcal{M} \stackrel{\simeq}{\longrightarrow}$ $\mathcal{M}$, see Definition 6.2. Since $J_{\mathcal{M}}$ equals $\operatorname{Id}_{\mathcal{M}}$ as a functor, we can view $\vartheta^{ \pm}$as isomorphisms of functors $\vartheta^{ \pm}: J_{\mathcal{M}}^{ \pm 1} \stackrel{\simeq}{\longrightarrow} D^{2}$.

The next result is proved in $\S 14$.

Proposition 6.10. The isomorphisms $\vartheta^{ \pm}: J_{\mathcal{M}}^{ \pm 1} \stackrel{\simeq}{\longrightarrow} D^{2}$ are monoidal.

Clearly Proposition 6.1 follows from Proposition 6.10.

6.3. The canonical monoidal isomorphism $\operatorname{Id}_{\mathcal{M}} \stackrel{\simeq}{\longrightarrow} D^{4}$ and the canonical doubletwist. Let $(\mathcal{M}, K, \beta)$ be a braided Grothendieck-Verdier category. In $\S 6.2$ we defined monoidal isomorphisms $\vartheta^{+}: J_{\mathcal{M}} \stackrel{\simeq}{\longrightarrow} D^{2}$ and $\vartheta^{-}: J_{\mathcal{M}}^{-1} \stackrel{\simeq}{\longrightarrow} D^{2}$.

Definition 6.11. We put $\gamma_{\mathcal{M}}=\vartheta^{+} \vartheta^{-}: \operatorname{Id}_{\mathcal{M}} \stackrel{\simeq}{\longrightarrow} D^{4}$ and call it the canonical monoidal isomorphism between $\operatorname{Id}_{\mathcal{M}}$ and $D^{4}$.

The next two remarks give alternative formulas for $\gamma_{\mathcal{M}}$.

Remark 6.12. One has $\gamma_{\mathcal{M}}=\vartheta^{-} \vartheta^{+}$. To see this, note that if $\mathcal{C}$ is any monoidal category and $c \in \mathcal{C}$ is isomorphic to $\mathbb{1}_{\mathcal{C}}$ then for each $f^{+}, f^{-} \in \operatorname{Hom}\left(\mathbb{1}_{\mathcal{C}}, c\right)$ the morphism $f^{+} \otimes f^{-}$: $\mathbb{1}_{\mathcal{C}} \longrightarrow c \otimes c$ equals $f^{-} \otimes f^{+}$. Now let $\mathcal{C}$ be the monoidal category of functors $\mathcal{M} \longrightarrow \mathcal{M}$, $c:=D^{2}, f^{ \pm}:=\vartheta^{ \pm}$(recall that $J_{\mathcal{M}}$ equals $\operatorname{Id}_{\mathcal{M}}$ as a functor). 
Remark 6.13. Clearly $\vartheta^{-}$defines a monoidal isomorphism $\left(\vartheta^{-}\right)^{(-1)}: D^{-2} \stackrel{\simeq}{\longrightarrow} J_{\mathcal{M}}$. One can check that $\gamma_{\mathcal{M}}$ is equal to the isomorphism $\operatorname{Id}_{\mathcal{M}} \stackrel{\simeq}{\longrightarrow} D^{4}$ corresponding to the composition $\vartheta^{+} \circ\left(\vartheta^{-}\right)^{(-1)}: D^{-2} \stackrel{\simeq}{\longrightarrow} D^{2}$. We do not use this fact in this article.

On the other hand, the isomorphism $\left(\vartheta^{+}\right)^{-1} \circ \vartheta^{-}: J_{\mathcal{M}}^{-1} \stackrel{\simeq}{\longrightarrow} J_{\mathcal{M}}$ defines a monoidal isomorphism $C_{\mathcal{M}}: \operatorname{Id}_{\mathcal{M}} \stackrel{\simeq}{\longrightarrow} J_{\mathcal{M}}^{2}$, i.e., a double-twist in the sense of Definition 6.5.

Definition 6.14. $C_{\mathcal{M}}$ is called the canonical double-twist of $(\mathcal{M}, K, \beta)$.

For each $X \in \mathcal{M}$, the isomorphisms $\gamma_{\mathcal{M}}: \operatorname{Id}_{\mathcal{M}} \stackrel{\simeq}{\longrightarrow} D^{4}$ and $C_{\mathcal{M}}: \operatorname{Id}_{\mathcal{M}} \stackrel{\simeq}{\longrightarrow} J_{\mathcal{M}}^{2}$ define isomorphisms $\gamma_{X}: X \stackrel{\simeq}{\longrightarrow} D^{4} X$ and $C_{X}: X \stackrel{\simeq}{\longrightarrow} X$ (recall that $J_{\mathcal{M}}$ equals $\operatorname{Id}_{\mathcal{M}}$ as a functor). By definition,

$$
C_{X}=\left(\vartheta_{X}^{+}\right)^{-1} \circ \vartheta_{X}^{-}
$$

Lemma 6.15. $\gamma_{X}=\vartheta_{D^{2} X}^{+} \circ \vartheta_{X}^{-}=D^{2}\left(\vartheta_{X}^{+}\right) \circ \vartheta_{X}^{-}=\vartheta_{D^{2} X}^{-} \circ \vartheta_{X}^{+}=D^{2}\left(\vartheta_{X}^{-}\right) \circ \vartheta_{X}^{+}$.

Proof. $\vartheta^{ \pm}: \operatorname{Id}_{\mathcal{M}} \stackrel{\simeq}{\longrightarrow} D^{2}$ is an isomorphism of functors, so for any $X, Y \in \mathcal{M}$ and any $f: X \longrightarrow Y$ one has $D^{2}(f) \circ \vartheta_{X}^{ \pm}=\vartheta_{Y}^{ \pm} \circ f$. Taking $Y=D^{2} X, f=\vartheta_{X}^{ \pm}$one gets $D^{2}\left(\vartheta_{X}^{+}\right)=\vartheta_{D^{2} X}^{+}$. Taking $Y=D^{2} X, f=\vartheta_{X}^{\mp}$ one gets $D^{2}\left(\vartheta_{X}^{\mp}\right) \circ \vartheta_{X}^{ \pm}=\vartheta_{D^{2} X}^{ \pm} \circ \vartheta_{X}^{\mp}$.

Remarks 6.16. (i) Combining formula (6.6) and Lemma 6.15 with formula (6.5) one sees that

$$
C_{D X}=D\left(C_{X}\right)
$$

and

$$
\gamma_{D X}=D\left(\gamma_{X}\right)^{-1}
$$

(ii) By Remark 6.6(ii), one has $C_{\mathbb{1}}=\mathrm{id}_{\mathbb{1}}$. By (6.7), this implies that

$$
C_{K}=\mathrm{id}_{K} \text {. }
$$

\section{Pivotal structures on Braided Grothendieck-Verdier Categories}

This section is closely related to [EMC10, Section 4]. We thank the referee for informing us about this fact.

7.1. Pivotal structures and twists. The notion of a pivotal structure on a (not necessarily braided) Grothendieck-Verdier category was introduced in Definition 5.1. Recall that by Proposition 5.7, a pivotal structure on a Grothendieck-Verdier category $(\mathcal{M}, K)$ is the same as a monoidal isomorphism $f: \operatorname{Id}_{\mathcal{M}} \stackrel{\simeq}{\longrightarrow} D^{2}$ such that $f_{K}: K \stackrel{\simeq}{\longrightarrow} D^{2} K$ is equal to the isomorphism (1.11). So by abuse of language, we often say that $f$ is a pivotal structure. Now suppose that $\mathcal{M}$ is equipped with a braiding $\beta$.

Proposition 7.1. Let $(\mathcal{M}, K, \beta)$ be a braided Grothendieck-Verdier category. Then the map $f \mapsto\left(\vartheta^{+}\right)^{-1} \circ f$ defines a bijection between the set of pivotal structures $f: \operatorname{Id}_{\mathcal{M}} \stackrel{\simeq}{\longrightarrow} D^{2}$ and the set of twists $\theta$ on $\mathcal{M}$ that satisfy $\theta_{K}=\mathrm{id}_{K}$. 
Proof. This follows immediately from Proposition 6.10 and Definition 6.5.

Remark 7.2. In $§ 5.1$ we defined a pivotal structure to be an isomorphism

$$
\psi_{X, Y}: \operatorname{Hom}(X \otimes Y, K) \stackrel{\simeq}{\longrightarrow} \operatorname{Hom}(Y \otimes X, K), \quad X, Y \in \mathcal{M},
$$

satisfying certain properties. It is easy to check that the relation between $\psi$ and the corresponding twist $\theta$ is as follows:

$$
\psi_{X, Y}=\left(\theta_{Y} \otimes \mathrm{id}_{X}\right)^{*} \circ \beta_{Y, X}^{*}: \operatorname{Hom}(X \otimes Y, K) \stackrel{\simeq}{\longrightarrow} \operatorname{Hom}(Y \otimes X, K) \stackrel{\simeq}{\longrightarrow} \operatorname{Hom}(Y \otimes X, K) .
$$

7.2. The involution on the set of pivotal structures. Let $(\mathcal{M}, K, \beta)$ be a braided Grothendieck-Verdier category. In the next proposition we define a canonical involution on the set of all pivotal structures on $(\mathcal{M}, K)$ or equivalently, on the set of twists $\theta$ on $(\mathcal{M}, \beta)$ such that $\theta_{K}=\mathrm{id}_{K}$. The fixed points of this involution correspond to ribbon structures (see Definition 8.1 and Corollary 8.3 below).

Proposition 7.3. Let $(\mathcal{M}, K, \beta)$ be a braided Grothendieck-Verdier category.

(i) For every twist $\theta: \operatorname{Id}_{\mathcal{M}} \stackrel{\simeq}{\longrightarrow} J_{\mathcal{M}}$ there is a unique twist $\theta^{\prime}: \operatorname{Id}_{\mathcal{M}} \stackrel{\simeq}{\longrightarrow} J_{\mathcal{M}}$ such that $\theta \theta^{\prime}: \operatorname{Id}_{\mathcal{M}} \stackrel{\simeq}{\longrightarrow} J_{\mathcal{M}}^{2}$ is equal to the canonical double-twist $C_{\mathcal{M}}$ from Definition 6.14 .

(ii) The map $\theta \mapsto \theta^{\prime}$ is an involution.

(iii) If $\theta_{K}=\mathrm{id}_{K}$ then $\theta_{K}^{\prime}=\mathrm{id}_{K}$.

(iv) If $\theta_{K}=\operatorname{id}_{K}$ then $\theta_{X}^{\prime}=D^{-1}\left(\theta_{D X}\right)$.

(v) Suppose that $\theta_{K}=\operatorname{id}_{K}$. Let $f: \operatorname{Id}_{\mathcal{M}} \stackrel{\simeq}{\longrightarrow} D^{2}$ and $f^{\prime}: \operatorname{Id}_{\mathcal{M}} \stackrel{\simeq}{\longrightarrow} D^{2}$ be the pivotal structures corresponding to $\theta$ and $\theta^{\prime}$ by Proposition 7.1. Then the isomorphism $f f^{\prime}: \operatorname{Id}_{\mathcal{M}} \stackrel{\simeq}{\longrightarrow} D^{4}$ equals the canonical isomorphism $\gamma_{\mathcal{M}}: \operatorname{Id}_{\mathcal{M}} \stackrel{\simeq}{\longrightarrow} D^{4}$ from Definition 6.11.

Remark 7.4. If $K \simeq \mathbb{1}$ then the condition $\theta_{K}=\mathrm{id}_{K}$ holds automatically because by Remark 6.6, $\theta_{\mathbb{1}}=\mathrm{id}_{\mathbb{1}}$.

Proof. Statements (i) and (ii) follow from Remarks 6.6(iii-iv). Statement (iii) follows from formula (6.9).

Let us prove (iv). By (6.6) and the definition of $\theta^{\prime}$, this amounts to showing that

$$
\theta_{X} \circ D^{-1}\left(\theta_{D X}\right)=\left(\vartheta_{X}^{+}\right)^{-1} \circ \vartheta_{X}^{-}, \quad X \in \mathcal{M}
$$

By Proposition 7.1, $\theta=\left(\vartheta^{+}\right)^{-1} \circ f$ for some pivotal structure $f: \operatorname{Id}_{\mathcal{M}} \stackrel{\simeq}{\longrightarrow} D^{2}$. Then for every $X \in \mathcal{M}$ one has $\theta_{X}=\left(\vartheta_{X}^{+}\right)^{-1} \circ f_{X}$. By formula $(5.5), D^{-1}\left(f_{D X}\right)=f_{X}^{-1}$, so by formula (6.5), $D^{-1}\left(\theta_{D X}\right)=f_{X}^{-1} \circ \vartheta_{X}^{-}$. Formula (7.1) follows.

Finally, we prove $(\mathrm{v})$. By definition, $f=\vartheta^{+} \circ \theta$ and

$$
f^{\prime}=\vartheta^{+} \circ \theta^{\prime}=\vartheta^{+} \circ\left(\vartheta^{+}\right)^{-1} \circ \vartheta^{-} \circ \theta^{-1}=\vartheta^{-} \circ \theta^{-1}
$$


(in the last equality we view $\theta^{-1}$ as an isomorphism $\operatorname{Id}_{\mathcal{M}} \stackrel{\simeq}{\longrightarrow} J_{\mathcal{M}}^{-1}$ ). Since $\theta$ belongs to the Bernstein center of $\mathcal{M}$ (recall that $J_{\mathcal{M}}=\operatorname{Id}_{\mathcal{M}}$ as a functor), we have

$$
f f^{\prime}=\left(\vartheta^{+} \circ \theta\right) \cdot\left(\vartheta^{-} \circ \theta^{-1}\right)=\vartheta^{+} \vartheta^{-}=\gamma_{\mathcal{M}} .
$$

\section{Ribbon GrothendiecK-Verdier CAtegories}

Definition 8.1. A ribbon structure on a braided Grothendieck-Verdier category $(\mathcal{M}, K, \beta)$ is a twist $\theta$ on $(\mathcal{M}, \beta)$ such that

$$
\theta_{X}=D^{-1}\left(\theta_{D X}\right) \quad \text { for all } X \in \mathcal{M} .
$$

A ribbon Grothendieck-Verdier category is a braided Grothendieck-Verdier category with a ribbon structure.

Lemma 8.2. A twist $\theta$ satisfies (8.1) if and only if $\theta_{K}=\mathrm{id}_{K}$ and $\theta^{\prime}=\theta$, where $\theta^{\prime}$ is defined in Proposition 7.3(i).

Proof. By Proposition 7.3(iv), we only have to show that the equality $\theta_{K}=\mathrm{id}_{K}$ follows from (8.1). This is clear because $K=D \mathbb{1}$ and by Remark 6.6(ii), $\theta_{\mathbb{1}}=\mathrm{id}_{\mathbb{1}}$.

Corollary 8.3. The correspondence between twists and pivotal structures (see Proposition 7.1) induces a bijection between ribbon structures on $(\mathcal{M}, K, \beta)$ and those pivotal structure $f: \operatorname{Id}_{\mathcal{M}} \stackrel{\simeq}{\longrightarrow} D^{2}$ that are invariant under the involution $f \mapsto f^{\prime}$ from Proposition $7.3(v)$.

Proof. This follows from Lemma 8.2 and Propositions 7.1-7.3.

Example 8.4. The r-category $\mathscr{D}_{G}(G)$ from Example 0.10 has a canonical ribbon structure, see [BD11, §A.5]. It corresponds (in the sense of Proposition 7.1) to the pivotal structure from Example 5.10. If the group $G$ is finite and the ground field $k$ is algebraically closed then $\mathscr{D}_{G}(G)$ is the derived category of the abelian category $\mathcal{A}$ formed by modules over the quantum double of the group algebra of $G$, and the above-mentioned ribbon structure on $\mathscr{D}_{G}(G)$ comes from the standard ribbon structure on $\mathcal{A}$. (The definition of the quantum double and the standard ribbon structure on $\mathcal{A}$ can be found, e.g., in [BK01, §3.2]).

Remark 8.5. The identity (8.1) holds if and only if for any $X, Y \in \mathcal{M}$ and $B: X \otimes Y \longrightarrow K$ one has

$$
B \circ\left(\operatorname{id}_{X} \otimes \theta_{Y}\right)=B \circ\left(\theta_{X} \otimes \operatorname{id}_{Y}\right) .
$$

Note that unlike (8.1), formula (8.2) makes sense in any braided category with a fixed object $K$ ( $K$ does not have to be dualizing and $\mathcal{M}$ does not have to be GrothendieckVerdier). We do not know whether condition (8.2) is really interesting in this generality. 


\section{Relation Between R-CAtegories and Grothendieck-Verdier CATEgories}

In this section we will use the notions of idempotent arrow, closed idempotent, and Hecke subcategory (see Definitions 2.4 and 2.6). Lemma 9.3 and Proposition 9.4 below answer the following question: which Grothendieck-Verdier categories can be realized as Hecke subcategories of r-categories? In order to formulate the answer, we will need

Lemma 9.1. Let $\mathcal{M}$ be a monoidal category and $\pi: \mathbb{1} \longrightarrow$ e an idempotent arrow in $\mathcal{M}$.

(a) The isomorphisms $e=\mathbb{1} \otimes e \stackrel{\pi \otimes \mathrm{id}_{e}}{\longrightarrow} e \otimes e$ and $e=e \otimes \mathbb{1} \stackrel{\mathrm{id}_{e} \otimes \pi}{\longrightarrow} e \otimes e$ are equal.

(b) If $u: e \otimes e \stackrel{\simeq}{\longrightarrow} e$ is the inverse of either of the two isomorphisms in $(a)$, then the pair $(e, u)$ is a unit object [BD11, Def. 2.1(3)] of eMe.

(c) If $\varpi: \mathbb{1} \longrightarrow e$ is any other idempotent arrow in $\mathcal{M}$, there is a unique automorphism $f: e \stackrel{\simeq}{\longrightarrow}$ e such that $f \circ \varpi=\pi$.

Proof. Part (a) is [BD11, Lemma 2.10], part (b) follows from [BD11, Lemma 2.18], and part (c) is [BD11, Corollary 2.40].

9.1. Hecke subcategories of r-categories. Let $\mathcal{M}$ be an r-category, and let $\pi: \mathbb{1} \longrightarrow e$ be an idempotent arrow in $\mathcal{M}$. We have a canonical identification $D^{2}(\mathbb{1}) \stackrel{\simeq}{\longrightarrow} \mathbb{1}$, so we may view $D^{2}(\pi)$ as a morphism $D^{2}(\pi): \mathbb{1} \longrightarrow D^{2}(e)$. Since $D^{2}$ has a natural monoidal structure, it follows that $D^{2}(\pi)$ is an idempotent arrow.

Next suppose that $D^{2}(e) \cong e$. By Lemma 9.1(c) there is a unique isomorphism $\varphi$ : $D^{2}(e) \stackrel{\simeq}{\longrightarrow} e$ such that $\varphi \circ D^{2}(\pi)=\pi$. Moreover, the Hecke subcategory $\mathcal{M}^{\prime}:=e \mathcal{M} e$ is a Grothendieck-Verdier category with dualizing object $K^{\prime}:=D e$, and the duality functor for $\left(\mathcal{M}^{\prime}, K^{\prime}\right)$ can be canonically identified with the restriction $\left.D\right|_{\mathcal{M}^{\prime}}$, using the isomorphisms ${ }^{14}$

$$
\operatorname{Hom}\left(X \otimes Y, K^{\prime}\right) \stackrel{\simeq}{\longrightarrow} \operatorname{Hom}(X \otimes Y, \mathbb{1}) \stackrel{\simeq}{\longrightarrow} \operatorname{Hom}(X, D Y), \quad X, Y \in \mathcal{M}^{\prime} .
$$

(where the first arrow is induced by $D \pi: K^{\prime} \longrightarrow D \mathbb{1}=\mathbb{1}$ ). We write $\mathbb{1}^{\prime}=e$ for the unit object $^{15}$ of $\mathcal{M}^{\prime}$, and we keep the notation $D$ for the duality functor of $\left(\mathcal{M}^{\prime}, K^{\prime}\right)$.

We define $f: K^{\prime} \longrightarrow \mathbb{1}^{\prime}$ to be the composition $K^{\prime}=D e \stackrel{D \pi}{\longrightarrow} D \mathbb{1}=\mathbb{1} \stackrel{\pi}{\longrightarrow} e=\mathbb{1}^{\prime}$.

9.2. Properties of the triple $\left(\mathcal{M}^{\prime}, K^{\prime}, f\right)$. We begin with the following

Remark 9.2. If $(\mathcal{M}, K)$ is any Grothendieck-Verdier category and $f: K \longrightarrow \mathbb{1}$ is a morphism in $\mathcal{M}$, then using the canonical identifications $D K \stackrel{\simeq}{\longrightarrow}$ and $D \mathbb{1} \stackrel{\simeq}{\longrightarrow}$, we can view $D f$ as a morphism $K \longrightarrow \mathbb{1}$.

Lemma 9.3. Let $\mathbb{1} \stackrel{\pi}{\longrightarrow}$ e be an idempotent arrow in an r-category $\mathcal{M}$, and let $\left(\mathcal{M}^{\prime}, K^{\prime}, f\right)$ be the corresponding triple constructed as in $\S 9.1$. Then

(a) $D f=f($ cf. Remark 9.2);

\footnotetext{
${ }^{14}$ See [BD11, Lemma A.50] for more details.

${ }^{15}$ Strictly speaking, we use the structure of a unit object on $e$ coming from Lemma 9.1(b).
} 
(b) for each $X \in \mathcal{M}^{\prime}$, the map $g \mapsto g \circ \pi$ is a bijection $\operatorname{Hom}\left(\mathbb{1}^{\prime}, X\right) \stackrel{\simeq}{\longrightarrow} \operatorname{Hom}(\mathbb{1}, X)$;

(c) for each $X \in \mathcal{M}^{\prime}$, the map $h \mapsto D \pi \circ h$ is a bijection $\operatorname{Hom}\left(X, K^{\prime}\right) \stackrel{\simeq}{\longrightarrow} \operatorname{Hom}(X, \mathbb{1})$.

Proof. (a) With our identifications, we have $D^{2}(\pi)=\pi$. Therefore $D f=D(\pi \circ D \pi)=$ $D^{2}(\pi) \circ D \pi=\pi \circ D \pi=f$.

(b) This follows from the fact [BD11, Prop. 2.22(a)] that the functor $Y \mapsto e \otimes Y$ is left adjoint to the inclusion functor $\mathcal{M}^{\prime}=e \mathcal{M} e \hookrightarrow \mathcal{M}$.

(c) This follows from (b) using the fact that $D$ is an anti-autoequivalence of $\mathcal{M}$.

\subsection{The inverse construction.}

Proposition 9.4. Let $\left(\mathcal{M}^{\prime}, K^{\prime}\right)$ be a Grothendieck-Verdier category with unit object $\mathbb{1}^{\prime}$ and duality functor $D$, and let $f: K^{\prime} \longrightarrow \mathbb{1}^{\prime}$ be a morphism such that $D f=f$ (cf. Remark 9.2). Then the triple $\left(\mathcal{M}^{\prime}, K^{\prime}, f\right)$ arises from a closed idempotent in an r-category by means of the construction described in $§ 9.1$.

The proof of Proposition 9.4 will be given in $\$ \S 15.1-15.3$. In fact, given $\left(\mathcal{M}^{\prime}, K^{\prime}\right)$ and $f$ we will construct there a concrete $r$-category $\mathcal{M}$ and a closed idempotent $e \in \mathcal{M}$ such that $\left(\mathcal{M}^{\prime}, K^{\prime}, f\right)$ arises from $(\mathcal{M}, e)$. One can characterize this pair $(\mathcal{M}, e)$ by a universal property, see Remark 15.5.

Remark 9.5. The assumption $D f=f$ in Proposition 9.4 is not satisfied automatically, see $\S 15.4$.

\section{Part 2. Proofs of the main results}

\section{Rigidity}

In this section we prove Proposition 3.4 and Proposition 1.5.

10.1. Recollections on rigid duals. Let $\mathcal{M}$ be a monoidal category and $\epsilon: A \otimes B \longrightarrow \mathbb{1}$ a morphism.

Definition 10.1. We say ${ }^{16}$ that $(A, \epsilon)$ is a left rigid dual of $B$ or that $(B, \epsilon)$ is a right rigid dual of $A$ if there exists $c: \mathbb{1} \longrightarrow B \otimes A$ such that the compositions

$$
A=A \otimes \mathbb{1} \stackrel{\mathrm{id}_{A} \otimes c}{\longrightarrow} A \otimes B \otimes A \stackrel{\epsilon \otimes \mathrm{id}_{A}}{\longrightarrow} \mathbb{1} \otimes A=A
$$

and

$$
B=\mathbb{1} \otimes B \stackrel{c \otimes \mathrm{id}_{B}}{\longrightarrow} B \otimes A \otimes B \stackrel{\mathrm{id}_{B} \otimes \epsilon}{\longrightarrow} B \otimes \mathbb{1}=B
$$

are equal to $\mathrm{id}_{A}$ and $\mathrm{id}_{B}$, respectively. An object of $\mathcal{M}$ is said to be rigid if it has a left rigid dual and a right one. $\mathcal{M}$ is said to be rigid if each of its objects is.

\footnotetext{
${ }^{16}$ Some authors use the opposite convention for "left" and "right". The advantage of our convention is that if $\mathcal{M}$ is the category of endofunctors of some category then the left dual is the same as a left adjoint functor.
} 
It is well known that the left or right rigid dual of an object $X \in \mathcal{M}$ is unique up to unique isomorphism. We denote the left rigid dual of $X$ by $X^{*}$ and the right one by ${ }^{*} X$. It is also known that in the situation of Definition 10.1 the morphism $c: \mathbb{1} \longrightarrow B \otimes A$ is unique. We will formulate a criterion for its existence, which goes back to [De90, JS93].

If $\mathcal{M}$ is a monoidal category and $X, Y \in \mathcal{M}$ are objects such that the functor $Z \mapsto$ $\operatorname{Hom}(X \otimes Z, Y)$ is representable, then, following [De90], we denote the representing object by $\underline{\operatorname{Hom}}(X, Y)$.

Proposition 10.2. Let $\mathcal{M}$ be a monoidal category and $\epsilon: A \otimes B \longrightarrow \mathbb{1}$ a morphism in $\mathcal{M}$. The following statements are equivalent:

(i) $(B, \epsilon)$ is a right rigid dual of $A$ (equivalently, $(A, \epsilon)$ is a left rigid dual of $B)$;

(ii) $\underline{\operatorname{Hom}}(A, Y)$ exists for each $Y \in \mathcal{M}$; moreover, the morphism $B \otimes Y \longrightarrow \underline{\operatorname{Hom}}(A, Y)$ that comes from $\epsilon \otimes \mathrm{id}_{Y}: A \otimes B \otimes Y \longrightarrow \mathbb{1} \otimes Y=Y$ is an isomorphism for every $Y \in \mathcal{M}$;

(ii') for all $Y, Z \in \mathcal{M}$, the map

$$
\operatorname{Hom}(Z, B \otimes Y) \longrightarrow \operatorname{Hom}(A \otimes Z, Y)
$$

that takes an element $f \in \operatorname{Hom}(Z, B \otimes Y)$ to the composition

$$
A \otimes Z \stackrel{\mathrm{id}_{A} \otimes f}{\longrightarrow} A \otimes B \otimes Y \stackrel{\epsilon \otimes \mathrm{id}_{Y}}{\longrightarrow} \mathbb{1} \otimes Y=Y
$$

is bijective;

(iii) $\underline{\operatorname{Hom}}(A, \mathbb{1})$ and $\underline{\operatorname{Hom}}(A, A)$ exist; in addition, the morphisms $B \longrightarrow \underline{\operatorname{Hom}}(A, \mathbb{1})$ $\underline{\text { and }} B \otimes A \longrightarrow \underline{\operatorname{Hom}}(A, A)$ defined in (ii) are isomorphisms;

(iii') the map (10.3) is bijective for $Y=\mathbb{1}, Z=B$ and for $Y=A, Z=\mathbb{1}$;

$\left(i i i^{\prime \prime}\right)$ the map (10.3) is injective for $Y=\mathbb{1}, Z=B$ and surjective for $Y=A, Z=\mathbb{1}$.

Remarks 10.3. (1) It is easy to see that $(i i) \Leftrightarrow\left(i i^{\prime}\right)$ and $(i i i) \Rightarrow\left(i i i^{\prime}\right)$. Tautologically, $(i i) \Rightarrow(i i i)$ and $\left(i i^{\prime}\right) \Rightarrow\left(i i i^{\prime}\right) \Rightarrow\left(i i i^{\prime \prime}\right)$.

(2) The equivalence between $(i i)$ and $(i)$ is proved in [De90, Prop. 2.3]. The equivalence between $\left(i i^{\prime}\right)$ and $(i)$ is stated in [JS93, p. 70]. So it remains to prove that $\left(i i i^{\prime \prime}\right) \Rightarrow(i)$.

Proof of the implication $\left(\right.$ iii $\left.^{\prime \prime}\right) \Rightarrow(i)$ in Proposition 10.2. Applying hypothesis $\left(i i^{\prime \prime}\right)$ with $Y=A$ and $Z=\mathbb{1}$, we see that there is a morphism $c: \mathbb{1} \longrightarrow B \otimes A$ such that the composition (10.1) equals $\mathrm{id}_{A}$. Now let $\alpha$ denote the composition (10.2). It remains to show that $\alpha=\mathrm{id}_{B}$. Using the fact that the composition (10.1) equals $\mathrm{id}_{A}$, it is easy to check that the composition

$$
A \otimes B \stackrel{\operatorname{id}_{A} \otimes \alpha}{\longrightarrow} A \otimes B \stackrel{\epsilon}{\longrightarrow} \mathbb{1}
$$

equals $\epsilon$. Thus the assumption of $\left(i i i^{\prime \prime}\right)$ with $Y=\mathbb{1}$ and $Z=B$ forces $\alpha=\mathrm{id}_{B}$. 


\subsection{Proof of Proposition 3.4.}

Proof. We will apply the equivalences (i) $\Leftrightarrow($ ii $) \Leftrightarrow($ iii) from Proposition 10.2.

First, recall that by Remark 1.1(iii), the existence of $\underline{\operatorname{Hom}}(A, Y)$ is automatic; namely,

$$
\underline{\operatorname{Hom}}(A, Y)=D^{-1}(D Y \otimes A),
$$

which can also be written as $\underline{\operatorname{Hom}}(A, Y)=D^{-1} A \odot Y$ by formula (3.1). In particular, $\underline{\operatorname{Hom}}(A, \mathbb{1})=D^{-1} A$.

So the condition that the morphism $B \longrightarrow \underline{\operatorname{Hom}}(A, \mathbb{1})$ is an isomorphism (see Proposition 10.2) is equivalent to condition (a) of Proposition 3.4. If it holds, the morphism $B \otimes Y \longrightarrow \underline{\operatorname{Hom}}(A, Y)$ from Proposition 10.2 can be considered as a morphism $B \otimes Y \longrightarrow$ $\underline{\operatorname{Hom}}(D B, Y)=B \odot Y$, and one checks that it equals the morphism $B \otimes Y \longrightarrow B \odot Y$ defined in $\S 3.1$.

Now applying the equivalence $(\mathrm{i}) \Leftrightarrow($ iii) from Proposition 10.2 we see that $(B, \epsilon)$ is a right rigid dual of $A$ if and only if (a) and (b) hold. Applying the equivalence (i) $\Leftrightarrow$ (ii) we see that in this case the canonical morphism $B \otimes Y \longrightarrow B \odot Y$ is an isomorphism for every $Y \in \mathcal{M}$. Now replacing $\otimes: \mathcal{M} \times \mathcal{M} \longrightarrow \mathcal{M}$ with the opposite tensor product and $B$ with $A$ we see that the canonical isomorphism $Y \otimes A \longrightarrow Y \odot A$ is an isomorphism for every $Y \in \mathcal{M}$.

\subsection{Proof of Proposition 1.5.}

Lemma 10.4. Let $(\mathcal{M}, K)$ be a Grothendieck-Verdier category. Then the canonical morphisms $\mathbb{1} \longrightarrow \underline{\operatorname{Hom}}(K, K)$ and $\mathbb{1} \longrightarrow \underline{\operatorname{Hom}^{\prime}}(K, K)$ are isomorphisms.

Here $\underline{\text { Hom }}$ and $\underline{\mathrm{Hom}}^{\prime}$ are the internal Hom's, see Remark 1.1(iii).

Proof. Use (1.5)-(1.8) and (1.9).

Now let us prove Proposition 1.5, which says that a dualizing object of a monoidal category is invertible if and only if it is rigid.

Proof. Any invertible object is rigid. Now suppose that a dualizing object $K$ of a monoidal category $\mathcal{M}$ is rigid. Let ${ }^{*} K$ (resp. $K^{*}$ ) be its right (resp. left) rigid dual. By Proposition 10.2(iii), ${ }^{*} K \otimes K \simeq \underline{\operatorname{Hom}}(K, K)$, so Lemma 10.4 shows that ${ }^{*} K \otimes K \simeq \mathbb{1}$. Similarly, $K \otimes K^{*} \simeq \mathbb{1}$.

\section{The MONOIDAL STRUCTURE ON $D^{2}$}

11.1. Proof of Proposition 4.2. We first make an obvious remark, then formulate its "categorification," and finally explain how to apply it to define a monoidal structure on $D^{2}: \mathcal{M} \longrightarrow \mathcal{M}$, which, in fact, equals the one defined by (4.4). 
11.1.1. Obvious remark. Let $A$ be an associative ring and let $N$ be an $(A, A)$-bimodule. Suppose that for some $n_{0} \in N$ the maps $A \rightarrow N$ defined by $a \mapsto n_{0} a$ and $a \mapsto a n_{0}$ are injections with the same image. Define the $\operatorname{map} \varphi: A \rightarrow A$ by the equality $a n_{0}=n_{0} \varphi(a)$. Then $\varphi$ is a ring automorphism.

11.1.2. Categorification: a way to construct monoidal auto-equivalences. Let $\mathcal{A}$ be a monoidal category and let $\mathcal{N}$ be an $(\mathcal{A}, \mathcal{A}$ )-bimodule category (i.e., we are given a monoidal functor from $\mathcal{A} \times \mathcal{A}^{\text {opp }}$ to the monoidal category of functors $\mathcal{N} \rightarrow \mathcal{N}$, where $\mathcal{A}^{\text {opp }}$ is the category $\mathcal{A}$ equipped with the opposite tensor product). Suppose that for some $n_{0} \in \mathcal{N}$ the functors $\mathcal{A} \rightarrow \mathcal{N}$ defined by $X \mapsto n_{0} \otimes X$ and $X \mapsto X \otimes n_{0}$ are fully faithful and have the same essential image. Then there exists an equivalence $\Phi: \mathcal{A} \stackrel{\sim}{\longrightarrow} \mathcal{A}$ such that one has isomorphisms $f_{X}: X \otimes n_{0} \stackrel{\simeq}{\longrightarrow} n_{0} \otimes \Phi(X)$ functorial in $X \in \mathcal{A}$; such a pair $(\Phi, f)$ is unique up to unique isomorphism. We claim that $\Phi$ has a canonical structure of monoidal equivalence. Namely, define $u_{X_{1}, X_{2}}: \Phi\left(X_{1} \otimes X_{2}\right) \stackrel{\simeq}{\longrightarrow} \Phi\left(X_{1}\right) \otimes \Phi\left(X_{2}\right)$ so that the diagram

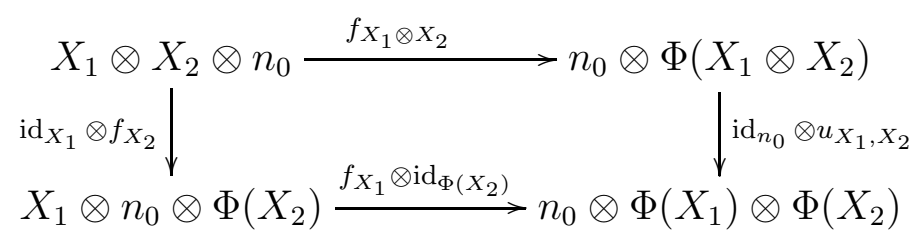

commutes. Similarly, we have a natural isomorphism $\Phi(\mathbb{1}) \stackrel{\simeq}{\longrightarrow}$. The isomorphisms $u_{X_{1}, X_{2}}$ are compatible with the associativity constraint and the unit constraints and thus define a structure of monoidal functor on $\Phi$.

11.1.3. Application. Take $\mathcal{A}=\mathcal{M}^{\circ}$, where $\mathcal{M}^{\circ}$ is the category dual to $\mathcal{M}$. Let $\mathcal{N}$ be the category of functors $F: \mathcal{M}^{\circ} \rightarrow\{$ Sets $\}$. It has a canonical structure of $\left(\mathcal{M}^{\circ}, \mathcal{M}^{\circ}\right)$-bimodule category such that $\left(m_{1} \otimes F \otimes m_{2}\right)(m)=F\left(m_{2} \otimes m \otimes m_{1}\right)$ (the associativity constraints of the bimodule category are the obvious ones). Let $\mathcal{Y}: \mathcal{M} \hookrightarrow \mathcal{N}$ be the Yoneda embedding and $n_{0}:=\mathcal{Y}(K) \in \mathcal{N}$. Using (1.1) and (1.2) one checks that $m \otimes n_{0}=\mathcal{Y}(D m)$ and $n_{0} \otimes m=\mathcal{Y}\left(D^{-1} m\right)$. So the above construction of a monoidal equivalence $\Phi: \mathcal{M}^{\circ} \stackrel{\sim}{\longrightarrow} \mathcal{M}^{\circ}$ is applicable and the functor $\mathcal{M} \stackrel{\sim}{\longrightarrow} \mathcal{M}$ corresponding to $\Phi$ equals $D^{2}$. Thus we get a structure of a monoidal functor on $D^{2}$.

11.1.4. Conclusion. One checks that the isomorphism $u_{X_{1}, X_{2}}: D^{2}\left(X_{1} \otimes X_{2}\right) \stackrel{\simeq}{\longrightarrow} D^{2}\left(X_{1}\right) \otimes$ $D^{2}\left(X_{2}\right)$ defined above equals the isomorphism (4.4) and that the isomorphism $\mathbb{1} \stackrel{\simeq}{\longrightarrow}$ $D^{2}(\mathbb{1})$ defined above equals the isomorphism (1.10). Proposition 4.2 follows.

11.2. A remark (to be used in $\S 14$ ). Suppose that in the situation of $\S 11.1 .2$ we have two pairs $(\Phi, f)$ and $(\tilde{\Phi}, \tilde{f})$, so the functors $\Phi$ and $\tilde{\Phi}$ are both monoidal. Let $\alpha: \tilde{\Phi} \stackrel{\simeq}{\longrightarrow} \Phi$ 
be the unique isomorphism such that the diagram

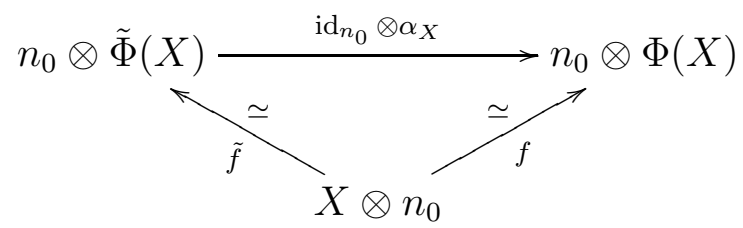

commutes. Then $\alpha$ is monoidal. To see this, compare (11.1) with a similar commutative square for $(\tilde{\Phi}, \tilde{f})$ by drawing a cube.

\section{Proof of Lemma 6.8}

12.1. An abstract lemma. In this subsection $\mathcal{M}$ is an abstract category rather than a monoidal one. The following lemma is used in the proof of Lemma 6.8 and Proposition 5.7.

Lemma 12.1. Let $\mathcal{M}$ be a category equipped with an anti-equivalence $D: \mathcal{M} \longrightarrow \mathcal{M}$. Let $S$ be the set of functorial families of bijections

$$
\gamma_{X, Y}: \operatorname{Hom}(X, D Y) \stackrel{\simeq}{\longrightarrow} \operatorname{Hom}(Y, D X), \quad X, Y \in \mathcal{M}
$$

(a) For each $\gamma \in S$ there is a unique isomorphism $\varphi^{\gamma}: D^{-1} \stackrel{\simeq}{\longrightarrow} D$ such that for all $X, Y \in \mathcal{M}$ the corresponding map $\operatorname{Hom}\left(Y, D^{-1} X\right) \stackrel{\simeq}{\longrightarrow} \operatorname{Hom}(Y, D X)$ is equal to the composition

$$
\operatorname{Hom}\left(Y, D^{-1} X\right) \stackrel{D}{\longrightarrow} \operatorname{Hom}(X, D Y) \stackrel{\gamma_{X, Y}}{\longrightarrow} \operatorname{Hom}(Y, D X) .
$$

The map $S \longrightarrow \operatorname{Isom}\left(D^{-1}, D\right)$ given by $\gamma \mapsto \varphi^{\gamma}$ is a bijection.

(b) Define an involution $\vee: S \stackrel{\simeq}{\longrightarrow} S$ by $\left(\gamma^{\vee}\right)_{X, Y}=\gamma_{Y, X}^{-1}$. Define a bijection

$$
\vee: \operatorname{Isom}\left(D^{-1}, D\right) \stackrel{\simeq}{\longrightarrow} \operatorname{Isom}\left(D^{-1}, D\right)
$$

by $\left(\varphi^{\vee}\right)_{X}:=D\left(\varphi_{D^{-1} X}^{-1}\right)$. Then

$$
\varphi^{\gamma^{\vee}}=\left(\varphi^{\gamma}\right)^{\vee} \quad \forall \gamma \in S
$$

In particular, $\vee$ is also an involution on $\operatorname{Isom}\left(D^{-1}, D\right)$ and can alternatively be defined by the formula $\left(\varphi^{\vee}\right)_{X}=D^{-1}\left(\varphi_{D X}^{-1}\right)$ for all $X \in \mathcal{M}$.

Proof. Yoneda's lemma implies statement (a) and gives explicit formulas for $\varphi^{\gamma}$ and $\left(\varphi^{\gamma}\right)^{-1}$. Namely, to obtain a formula for $\varphi_{X}^{\gamma}$, where $X \in \mathcal{M}$, apply the composition (12.1) for $Y=D^{-1} X$ to $\operatorname{id}_{D^{-1} X} \in \operatorname{Hom}\left(D^{-1} X, D^{-1} X\right)$; to obtain a formula for $\left(\varphi_{X}^{\gamma}\right)^{-1}$, consider the composition (12.1) for $Y=D X$ and apply its inverse to $\operatorname{id}_{D X} \in \operatorname{Hom}(D X, D X)$. Thus

$$
\varphi_{X}^{\gamma}=\gamma_{X, D^{-1} X}\left(\operatorname{id}_{X}\right)
$$

and

$$
\left(\varphi_{X}^{\gamma}\right)^{-1}=D^{-1}\left(\gamma_{X, D X}^{-1}\left(\operatorname{id}_{D X}\right)\right)
$$


Now let us deduce (12.2) from (12.3)-(12.4). By (12.3), $\varphi_{X}^{\gamma^{\vee}}=\gamma_{D^{-1} X, X}^{-1}\left(\mathrm{id}_{D^{-1} X}\right)$. Comparing this with (12.4), we see that $\varphi_{X}^{\gamma^{\vee}}=D\left(\left(\varphi_{D^{-1} X}^{\gamma}\right)^{-1}\right)$, which is equivalent to (12.2). The remaining assertions of (b) follow at once.

Corollary 12.2. Let $\gamma$ and $\varphi^{\gamma}$ be as in Lemma 12.1. For brevity, set $\varphi:=\varphi^{\gamma}$. Then the following properties are equivalent:

(i) $\gamma_{X, Y} \circ \gamma_{Y, X}=$ id for all $X, Y \in \mathcal{M}$.

(ii) $\varphi_{X}=D\left(\varphi_{D^{-1} X}\right)^{-1}$ for all $X \in \mathcal{M}$;

Proof. Property (i) means that $\gamma^{\vee}=\gamma$. Property (ii) means that $\varphi^{\vee}=\varphi$. So (i) $\Leftrightarrow$ (ii) by Lemma 12.1(b).

12.2. Proof of Lemma 6.8. Let $(\mathcal{M}, K, \beta)$ be a braided Grothendieck-Verdier category and $D: \mathcal{M} \stackrel{\sim}{\longrightarrow} \mathcal{M}$ the duality functor. Let $\beta^{ \pm}$be as in Lemma 6.8. Apply Lemma 12.1 to the functorial families of isomorphisms

$$
\gamma_{X, Y}^{ \pm}: \operatorname{Hom}(X, D Y) \stackrel{\simeq}{\longrightarrow} \operatorname{Hom}(Y, D X), \quad X, Y \in \mathcal{M},
$$

induced by the pullback maps

$$
\left(\beta_{Y, X}^{ \pm}\right)^{*}: \operatorname{Hom}(X \otimes Y, K) \stackrel{\simeq}{\longrightarrow} \operatorname{Hom}(Y \otimes X, K) .
$$

The isomorphism $\varphi^{\gamma^{ \pm}}$from Lemma 12.1(a) equals the isomorphism $\varphi^{ \pm}$from Lemma 6.8. With the notation of Lemma 12.1(b), $\left(\gamma^{ \pm}\right)^{\vee}=\gamma^{\mp}$. Thus the second assertion of Lemma 6.8 is equivalent to (12.2).

To prove the first assertion, note that the composition

$$
\operatorname{Hom}\left(D^{2} Y, D X\right) \stackrel{D^{-1}}{\longrightarrow} \operatorname{Hom}(X, D Y) \stackrel{\gamma_{X, Y}^{ \pm}}{\longrightarrow} \operatorname{Hom}(Y, D X)
$$

equals $\left(\vartheta_{Y}^{ \pm}\right)^{*}: \operatorname{Hom}\left(D^{2} Y, D X\right) \stackrel{\simeq}{\longrightarrow} \operatorname{Hom}(Y, D X)$ by Definition 6.7. Hence the map

$$
\operatorname{Hom}\left(Y, D^{-1} X\right) \longrightarrow \operatorname{Hom}(Y, D X)
$$

induced by $\varphi_{X}^{ \pm}: D^{-1} X \longrightarrow D X$ is equal to the composition

$$
\operatorname{Hom}\left(Y, D^{-1} X\right) \stackrel{D^{2}}{\longrightarrow} \operatorname{Hom}\left(D^{2} Y, D X\right) \stackrel{\left(\vartheta_{Y}^{ \pm}\right)^{*}}{\longrightarrow} \operatorname{Hom}(Y, D X) \text {; }
$$

more explicitly, $\varphi_{X}^{ \pm} \circ f=\left(D^{2} f\right) \circ \vartheta_{Y}^{ \pm}$for all $f \in \operatorname{Hom}\left(Y, D^{-1} X\right)$. Taking $X=D Y$ and $f=\operatorname{id}_{Y}$ yields $\vartheta_{Y}^{ \pm}=\varphi_{D Y}^{ \pm}$, as claimed.

\section{Proof of Proposition 5.7}

Throughout this section we fix a Grothendieck-Verdier category $(\mathcal{M}, K)$ together with a functorial family of isomorphisms

$$
\psi_{X, Y}: \operatorname{Hom}(X \otimes Y, K) \stackrel{\simeq}{\longrightarrow} \operatorname{Hom}(Y \otimes X, K), \quad X, Y \in \mathcal{M},
$$

and let $f: \operatorname{Id}_{\mathcal{M}} \stackrel{\simeq}{\longrightarrow} D^{2}$ be the corresponding isomorphism (see Lemma 5.6). 
13.1. Formulating the lemmas. The following lemmas will be proved in $§ 13.3-13.5$.

Lemma 13.1. With the notation above, $f$ is monoidal if and only if

$$
\psi_{X \otimes Y, Z} \circ \psi_{Y \otimes Z, X}=\psi_{Y, Z \otimes X} \quad \forall X, Y, Z \in \mathcal{M} .
$$

Lemma 13.2. Identity (5.3) holds if and only if $f_{D X}=D\left(f_{X}\right)^{-1}$ for all $X \in \mathcal{M}$.

Lemma 13.3. The following conditions are equivalent:

(a) $f_{K}: K \stackrel{\simeq}{\longrightarrow} D^{2} K$ equals the isomorphism (1.11);

(b) one has

$$
\psi_{\mathbb{1}, X}=\mathrm{id} \quad \text { for all } X \in \mathcal{M} \text {; }
$$

(c) $\psi_{\mathbb{1}, K}: \operatorname{Hom}(K, K) \rightarrow \operatorname{Hom}(K, K)$ maps $\operatorname{id}_{K}$ to itself.

13.2. Deducing Proposition 5.7 from the lemmas. Assume that $\psi$ is a pivotal structure. Identities (5.2)-(5.3) imply (13.1), so $f$ is monoidal by Lemma 13.1. Next, Lemma 13.2 shows that $f_{D X}=D\left(f_{X}\right)^{-1}$ for all $X \in \mathcal{M}$. Taking $X=\mathbb{1}$, we obtain $f_{K}=D\left(f_{\mathbb{1}}\right)^{-1}$. Since $f$ is monoidal, $f_{\mathbb{1}}$ is equal to the isomorphism (1.10), and therefore $f_{K}$ is equal to the isomorphism (1.11). Thus $f$ satisfies conditions (i)-(ii) of Proposition 5.7 and also satisfies (5.5).

Now suppose that $f$ satisfies conditions (i)-(ii) of Proposition 5.7. Then (13.1) and (13.2) hold by Lemmas 13.1 and 13.3. Setting $Y=\mathbb{1}$ in (13.1) and using (13.2) we see that

$$
\psi_{X, Z} \circ \psi_{Z, X}=\mathrm{id}
$$

Clearly (13.1) and (13.3) imply that $\psi$ is a pivotal structure, see Definition 5.1.

13.3. Proof of Lemma 13.1. Fix $X, Y, Z \in \mathcal{M}$. According to the definition of the correspondence between $f$ and $\psi$ (see Lemma 5.6), the isomorphism

$$
\psi_{Y, Z \otimes X}: \operatorname{Hom}(Y \otimes Z \otimes X, K) \stackrel{\simeq}{\longrightarrow} \operatorname{Hom}(Z \otimes X \otimes Y, K)
$$

is equal to the composition of

$$
\operatorname{Hom}(Y \otimes Z \otimes X, K) \stackrel{(1.3)}{\longrightarrow} \operatorname{Hom}\left(D^{2}(Z \otimes X) \otimes Y, K\right)
$$

and

$$
\left(f_{Z \otimes X} \otimes \operatorname{id}_{Y}\right)^{*}: \operatorname{Hom}\left(D^{2}(Z \otimes X) \otimes Y, K\right) \stackrel{\simeq}{\longrightarrow} \operatorname{Hom}(Z \otimes X \otimes Y, K) .
$$

Similarly, the isomorphism

$$
\psi_{X \otimes Y, Z} \circ \psi_{Y \otimes Z, X}: \operatorname{Hom}(Y \otimes Z \otimes X, K) \stackrel{\simeq}{\longrightarrow} \operatorname{Hom}(Z \otimes X \otimes Y, K)
$$

is equal to the composition of the isomorphisms

$$
\operatorname{Hom}(Y \otimes Z \otimes X, K) \stackrel{(1.3)}{\longrightarrow} \operatorname{Hom}\left(D^{2} X \otimes Y \otimes Z, K\right) \stackrel{(1.3)}{\longrightarrow} \operatorname{Hom}\left(D^{2} Z \otimes D^{2} X \otimes Y, K\right)
$$

followed by

$$
\left(f_{Z} \otimes f_{X} \otimes \mathrm{id}_{Y}\right)^{*}: \operatorname{Hom}\left(D^{2} Z \otimes D^{2} X \otimes Y, K\right) \stackrel{\simeq}{\longrightarrow} \operatorname{Hom}(Z \otimes X \otimes Y, K) .
$$


So property (13.1) is equivalent to the commutativity of the outer pentagon in the diagram

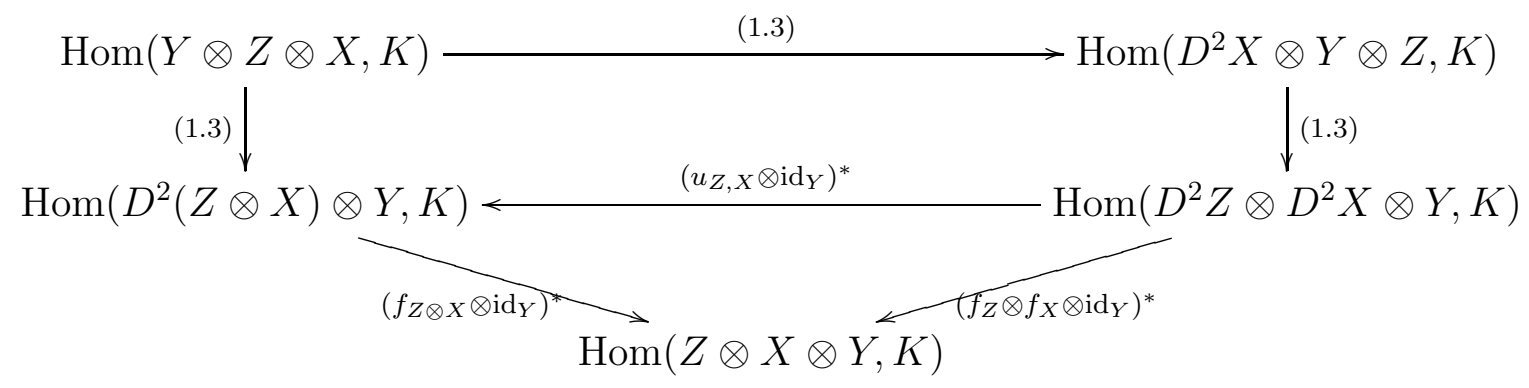

In this diagram $u_{Z, X}: D^{2}(Z \otimes X) \stackrel{\simeq}{\longrightarrow} D^{2} Z \otimes D^{2} X$ is the isomorphism defining the monoidal structure on $D^{2}$. By the definition of $u$ (which was given immediately before Proposition 4.2), the top square of the diagram commutes. So the commutativity of the outer pentagon is equivalent to that of the bottom triangle. The latter is equivalent to $f$ being monoidal (see Lemma 4.1).

13.4. Proof of Lemma 13.2. Let $\gamma$ denote the functorial family of bijections

$$
\gamma_{X, Y}: \operatorname{Hom}(X, D Y) \stackrel{\simeq}{\longrightarrow} \operatorname{Hom}(X \otimes Y, K) \stackrel{\psi_{X, Y}}{\longrightarrow} \operatorname{Hom}(Y \otimes X, K) \stackrel{\simeq}{\longrightarrow} \operatorname{Hom}(Y, D X)
$$

where the first and third arrows come from (1.1). Let $\varphi$ be as in Corollary 12.2. Comparing Lemma 12.1(a) with Lemma 5.6 we see that $\varphi_{X}=f_{D^{-1} X}$. So by Corollary 12.2, the condition

$$
\psi_{X, Y} \circ \psi_{Y, X}=\text { id } \quad \forall X, Y \in \mathcal{M}
$$

is equivalent to the condition

$$
f_{D^{-1} X}=D\left(f_{D^{-2} X}\right)^{-1} \quad \forall X \in \mathcal{M} .
$$

The latter condition holds if and only if $f_{D X}=D\left(f_{X}\right)^{-1}$ for all $X \in \mathcal{M}$, Q.E.D.

13.5. Proof of Lemma 13.3. The isomorphism $\psi_{\mathbb{1}, X}: \operatorname{Hom}(X, K) \stackrel{\simeq}{\longrightarrow} \operatorname{Hom}(X, K)$ is functorial in $X \in \mathcal{M}$, so (b) $\Leftrightarrow(\mathrm{c})$ by Yoneda's lemma. To prove that (a) $\Leftrightarrow(\mathrm{c})$, it suffices to show that $\psi_{\mathbb{1}, K}=\gamma \circ f_{K}$, where $\gamma: D^{2} K \stackrel{\simeq}{\longrightarrow} K$ is inverse to (1.11). Diagram (5.4) for $X=\mathbb{1}$ and $Y=K$ tells us that $\psi_{\mathbb{1}, K}=\gamma^{\prime} \circ f_{K}$, where $\gamma^{\prime} \in \operatorname{Hom}\left(D^{2} K, K\right)$ is the image of $\operatorname{id}_{K} \in \operatorname{Hom}(\mathbb{1} \otimes K, K)$ under the isomorphism (1.3). But $\gamma^{\prime}=\gamma$ by Remark 1.1(v).

\section{Proof of Proposition 6.10}

The idea of the proof is to use the relation between monoidal auto-equivalences and bimodule categories explained in $\S 11.1$.

Just as in $\S 11.1 .3$, we let $\mathcal{N}$ denote the category of functors $\mathcal{M}^{\circ} \longrightarrow$ Sets, we write $\mathcal{Y}: \mathcal{M} \hookrightarrow \mathcal{N}$ for the Yoneda embedding and put $n_{0}:=\mathcal{Y}(K) \in \mathcal{N}$. We equip $\mathcal{N}$ with the $\left(\mathcal{M}^{\circ}, \mathcal{M}^{\circ}\right)$-bimodule structure from $\S 11$.1.3. For this structure, $\left(X \otimes n_{0}\right)(Z)=$ 
$\operatorname{Hom}(Z \otimes X, K)$ and $\left(n_{0} \otimes X\right)(Z)=\operatorname{Hom}(X \otimes Z, K)$ for all $X, Z \in \mathcal{M}$. In $\S 11.1 .3$ we defined the monoidal structure on $D^{2}$ using the isomorphism

$$
X \otimes n_{0} \stackrel{\simeq}{\longrightarrow} n_{0} \otimes D^{2} X, \quad X \in \mathcal{M},
$$

which comes from (1.3). On the other hand, the isomorphisms

$$
\left(\beta_{X, Z}^{ \pm}\right)^{*}: \operatorname{Hom}(Z \otimes X, K) \stackrel{\simeq}{\longrightarrow} \operatorname{Hom}(X \otimes Z, K), \quad X, Z \in \mathcal{M},
$$

define isomorphisms

$$
f_{X}^{ \pm}: X \otimes n_{0} \stackrel{\simeq}{\longrightarrow} n_{0} \otimes X, \quad X \in \mathcal{M} .
$$

By $§ 11.1 .2$, each of the isomorphisms (14.2) defines a monoidal structure $s^{ \pm}$on the identity functor $\operatorname{Id}_{\mathcal{M}}$. By $\S 11.2$, we have a canonical monoidal isomorphism $\alpha^{ \pm}:\left(\operatorname{Id}_{\mathcal{M}}, s^{ \pm}\right) \stackrel{\simeq}{\longrightarrow} D^{2}$. Thus to prove Proposition 6.10 it suffices to prove the following lemma.

Lemma 14.1. (i) The isomorphism $\alpha^{ \pm}: \operatorname{Id}_{\mathcal{M}} \stackrel{\simeq}{\longrightarrow} D^{2}$ defined above equals the isomorphism $\vartheta^{ \pm}: \operatorname{Id}_{\mathcal{M}} \stackrel{\simeq}{\longrightarrow} D^{2}$ from Definition 6.7 .

(ii) The monoidal structure on $\operatorname{Id}_{\mathcal{M}}$ induced by the isomorphisms $f_{X}^{+}$(resp., $f_{X}^{-}$) is equal to the monoidal structure defined by (6.1) (resp., (6.2)).

Proof. To prove (i), we have to show that for each $X \in \mathcal{M}$, the diagram

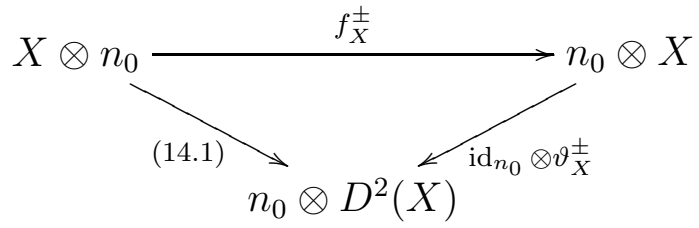

commutes; here the bottom left arrow is the isomorphism (14.1). This is a diagram of functors; evaluating them on a test object $Y \in \mathcal{M}$, we get the diagram

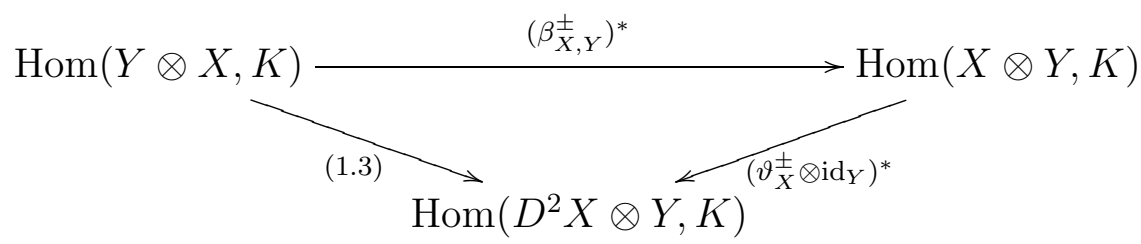

which commutes by Definition 6.7.

Statement (ii) of the lemma is equivalent to the following easy fact about braided monoidal categories: if $X, Y, Z \in \mathcal{M}$, then the square

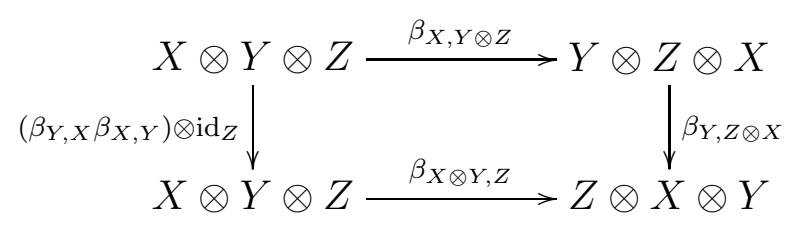


commutes. To verify this fact, note that by the hexagon axiom, the diagram

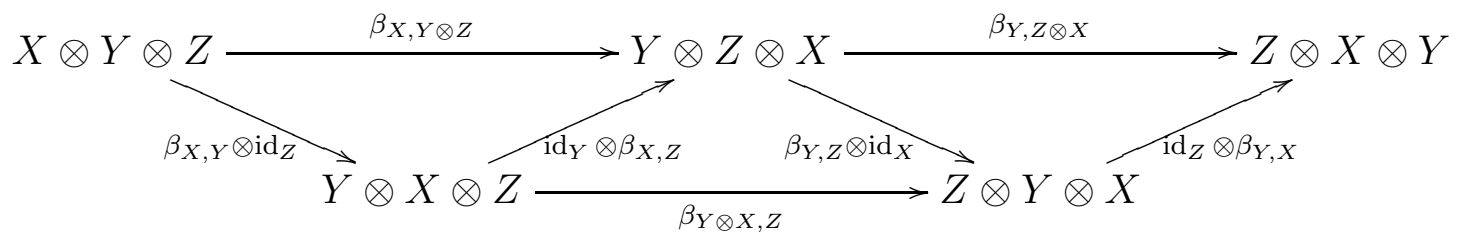

commutes. Moreover, the functoriality of $\beta$ implies that

$$
\left(\operatorname{id}_{Z} \otimes \beta_{Y, X}\right) \circ \beta_{Y \otimes X, Z}=\beta_{X \otimes Y, Z} \circ\left(\beta_{Y, X} \otimes \operatorname{id}_{Z}\right),
$$

which implies that (14.3) commutes and proves statement (ii).

\section{From Grothendieck-Verdier categories to R-CAtegories}

In $\S \S 15.1-15.3$ we prove Proposition 9.4. In $\S 15.4$ we give an example showing that in Proposition 9.4 the condition $D f=f$ does not hold automatically.

To prove Proposition 9.4, we provide a right inverse for the construction from $\S 9.1$. Namely, given a Grothendieck-Verdier category $\left(\mathcal{M}^{\prime}, K^{\prime}\right)$ and a morphism $f: K^{\prime} \longrightarrow \mathbb{1}^{\prime}$ such that $D f=f$, we construct an r-category $\mathcal{M}$ and a closed idempotent $e \in \mathcal{M}$ (this is done in two steps: in $\S 15.1$ we construct $\mathcal{M}$ as an abstract category, and in $\S 15.2$ we define the monoidal structure on $\mathcal{M}$ ). Then we show in Lemmas 15.4-15.6 that the pair $(\mathcal{M}, e)$ has the properties required in Proposition 9.4 (in particular, we prove that the monoidal category $\mathcal{M}$ is an r-category).

15.1. $\mathcal{M}$ as an abstract category. In this subsection we work with abstract categories rather than monoidal ones. For convenience, we preserve the same notation as above: namely, we consider a category $\mathcal{M}^{\prime}$ together with objects $K^{\prime}, \mathbb{1}^{\prime} \in \mathcal{M}^{\prime}$ and a morphism $f: K^{\prime} \longrightarrow \mathbb{1}^{\prime}$. However, we make no assumptions about $K^{\prime}, \mathbb{1}^{\prime}, f$.

Definition 15.1 . Let $(\mathcal{M}, \iota, \mathbb{1}, \delta, \pi)$ be the universal ${ }^{17}$ datum consisting of a category $\mathcal{M}$, a functor $\iota: \mathcal{M}^{\prime} \longrightarrow \mathcal{M}$ and a commutative triangle

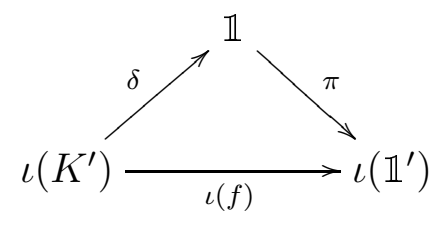

in $\mathcal{M}$ (note that $\mathcal{M}$ is determined uniquely up to isomorphism of categories).

Lemma 15.2. Let $(\mathcal{M}, \iota, \mathbb{1}, \delta, \pi)$ be as in the definition above. Then

(i) $\iota$ is fully faithful;

\footnotetext{
${ }^{17}$ I.e., initial as an object of the (1-)category of all such data, with the obvious notion of morphism.
} 
(ii) for each $X \in \mathcal{M}^{\prime}$, the maps

$$
\operatorname{Hom}_{\mathcal{M}}\left(\iota(X), \iota\left(K^{\prime}\right)\right) \longrightarrow \operatorname{Hom}_{\mathcal{M}}(\iota(X), \mathbb{1}), \quad g \mapsto \delta \circ g,
$$

and

$$
\operatorname{Hom}_{\mathcal{M}}\left(\iota\left(\mathbb{1}^{\prime}\right), \iota(X)\right) \longrightarrow \operatorname{Hom}_{\mathcal{M}}(\mathbb{1}, \iota(X)), \quad g \mapsto g \circ \pi,
$$

are bijective.

(iii) the map

$$
\operatorname{Hom}\left(\iota\left(K^{\prime}\right), \iota\left(\mathbb{1}^{\prime}\right)\right) \longrightarrow \operatorname{Hom}(\mathbb{1}, \mathbb{1}), \quad g \mapsto \delta \circ g \circ \pi
$$

is injective with image $\operatorname{Hom}(\mathbb{1}, \mathbb{1}) \backslash\left\{\mathrm{id}_{\mathbb{1}}\right\}$.

Proof. We first construct a datum $(\mathcal{M}, \iota, \mathbb{1}, \delta, \pi)$ for which properties (i)-(iii) are obvious and then check that this datum is universal.

The class of objects $O b(\mathcal{M})$ is defined to be the disjoint union of $O b\left(\mathcal{M}^{\prime}\right)$ and a oneelement set $\{\mathbb{1}\}$. Define maps $\Phi, \Psi: O b(\mathcal{M}) \longrightarrow O b\left(\mathcal{M}^{\prime}\right)$ as follows:

$$
\Phi(\mathbb{1})=\mathbb{1}^{\prime}, \quad \Psi(\mathbb{1})=K^{\prime}, \quad \Phi(X)=\Psi(X)=X \quad \forall X \in O b\left(\mathcal{M}^{\prime}\right) .
$$

For all $X \in O b(\mathcal{M})$ let $f_{X}: \Psi(X) \longrightarrow \Phi(X)$ be the morphism in $\mathcal{M}^{\prime}$ given by

$$
f_{\mathbb{1}}=f, \quad f_{X}=\mathrm{id}_{X} \quad \text { for } \quad X \in O b\left(\mathcal{M}^{\prime}\right) .
$$

For $X, Y \in O b(\mathcal{M})$, set $\operatorname{hom}(X, Y)=\operatorname{Hom}_{\mathcal{M}^{\prime}}(\Phi(X), \Psi(Y))$. Given $X, Y, Z \in O b(\mathcal{M})$ and $u \in \operatorname{hom}(X, Y)=\operatorname{Hom}_{\mathcal{M}^{\prime}}(\Phi(X), \Psi(Y))$ and $v \in \operatorname{hom}(Y, Z)=\operatorname{Hom}_{\mathcal{M}^{\prime}}(\Phi(Y), \Psi(Z))$, set $v \tilde{o} u:=v \circ f_{Y} \circ v \in \operatorname{hom}(X, Z)=\operatorname{Hom}_{\mathcal{M}^{\prime}}(\Phi(X), \Psi(Z))$. It is evident that o defines an associative composition operation on the sets $\operatorname{hom}(X, Y)$.

Now add to $\mathcal{M}$ one more morphism $\mathbb{1} \longrightarrow \mathbb{1}$, denoted by id $\mathbb{1}_{\mathbb{1}}$, and extend the operation o by setting $\operatorname{id}_{\mathbb{1}} \tilde{o} u=u$ and $v \tilde{o} \mathrm{id}_{\mathbb{1}}=v$ whenever these compositions make sense. Then $\mathcal{M}$ becomes a category. By construction, $\mathcal{M}^{\prime}$ is a full subcategory of $\mathcal{M}$. Let $\iota: \mathcal{M}^{\prime} \hookrightarrow \mathcal{M}$ be the inclusion functor, let $\delta \in \operatorname{hom}\left(K^{\prime}, \mathbb{1}\right)$ correspond to $\operatorname{id}_{K^{\prime}} \in \operatorname{Hom}_{\mathcal{M}^{\prime}}\left(K^{\prime}, K^{\prime}\right)$, and let $\pi \in \operatorname{hom}\left(\mathbb{1}, \mathbb{1}^{\prime}\right)$ correspond to $\operatorname{id}_{\mathbb{1}^{\prime}} \in \operatorname{Hom}_{\mathcal{M}^{\prime}}\left(\mathbb{1}^{\prime}, \mathbb{1}^{\prime}\right)$.

The datum $(\mathcal{M}, \iota, \mathbb{1}, \delta, \pi)$ clearly has properties (i)-(iii). It remains to check that this datum is universal, i.e., given another datum $(\overline{\mathcal{M}}, \bar{\iota}, \overline{\mathbb{1}}, \bar{\delta}, \bar{\pi})$ there is a unique functor $F: \mathcal{M} \longrightarrow \overline{\mathcal{M}}$ such that

$$
\begin{aligned}
& \left.F\right|_{\mathcal{M}^{\prime}}=\bar{\iota}, \quad F(\mathbb{1})=\overline{\mathbb{1}}, \\
& F(\delta)=\bar{\delta}, \quad F(\pi)=\bar{\pi} .
\end{aligned}
$$

If such $F$ exists then one should have

$$
\begin{gathered}
F(\delta \tilde{\circ} g)=\bar{\delta} \circ \bar{\iota}(g) \quad \forall g \in \operatorname{Hom}\left(X, K^{\prime}\right), X \in \mathcal{M}^{\prime} ; \\
F(g \tilde{\circ} \pi)=\bar{\iota}(g) \circ \bar{\pi} \quad \forall g \in \operatorname{Hom}(\mathbb{1}, X), X \in \mathcal{M}^{\prime} ; \\
F(\delta \tilde{\circ} g \tilde{\circ} \pi)=\bar{\delta} \circ \bar{\iota}(g) \circ \bar{\pi} \quad \forall g \in \operatorname{Hom}\left(K^{\prime}, \mathbb{1}\right) .
\end{gathered}
$$

Since $\mathcal{M}$ has properties (i)-(iii) the action of $F$ on objects and morphisms is uniquely determined by (15.1) and (15.2)-(15.4). It is easy to check that the action of $F$ on morphisms defined by (15.1)-(15.4) agrees with composition. 
15.2. $\mathcal{M}$ as a monoidal category. In this subsection we assume that $\mathcal{M}^{\prime}$ is a monoidal category with unit object $\mathbb{1}^{\prime}$, and that $\left(K^{\prime}, f\right)$ is a pair consisting of an object $K^{\prime} \in \mathcal{M}^{\prime}$ and a morphism $f: K^{\prime} \longrightarrow \mathbb{1}^{\prime}$. Let $(\mathcal{M}, \iota, \mathbb{1}, \delta, \pi)$ be as in Definition 15.1. By Lemma $15.2, \iota$ is fully faithful, so we will view $\mathcal{M}^{\prime}$ as a full subcategory of $\mathcal{M}$ and omit the symbol $\iota$ from now on.

Lemma 15.3. Suppose that the following diagram commutes:

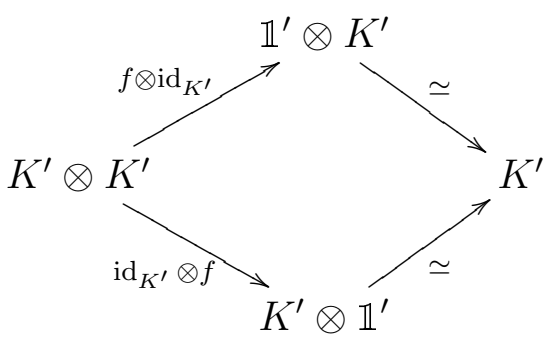

Then there is a unique way to extend the monoidal structure $\otimes: \mathcal{M}^{\prime} \times \mathcal{M}^{\prime} \longrightarrow \mathcal{M}^{\prime}$ to a bifunctor $\otimes: \mathcal{M} \times \mathcal{M} \longrightarrow \mathcal{M}$ so that the following properties are satisfied:

(1) the functors $X \longmapsto \mathbb{1} \otimes X$ and $X \longmapsto X \otimes \mathbb{1}$ are equal to the identity functor on $\mathcal{M}$;

(2) for each $X \in \mathcal{M}^{\prime}$, the morphisms

$$
X=\mathbb{1} \otimes X \stackrel{\pi \otimes \operatorname{id}_{X}}{\longrightarrow} \mathbb{1}^{\prime} \otimes X \quad \text { and } \quad X=X \otimes \mathbb{1} \stackrel{\operatorname{id}_{X} \otimes \pi}{\longrightarrow} X \otimes \mathbb{1}^{\prime}
$$

are equal to the isomorphisms $X \stackrel{\simeq}{\longrightarrow} \mathbb{1}^{\prime} \otimes X$ and $X \stackrel{\simeq}{\longrightarrow} X \otimes \mathbb{1}^{\prime}$ that come from the structure of unit object on $\mathbb{1}^{\prime} \in \mathcal{M}^{\prime}$.

Furthermore, there is a unique way to extend the associativity constraint for $\otimes$ on $\mathcal{M}^{\prime}$ to an associativity constraint for the bifunctor $\otimes$ on $\mathcal{M}$ so that $(\mathcal{M}, \otimes, \mathbb{1})$ becomes a monoidal category with trivial unit constraints.

Proof. The universal property of $\mathcal{M}$ implies that there is a unique way to define $X \otimes Y$ as a functor of $Y \in \mathcal{M}$ for a fixed $X \in \mathcal{M}$, and as a functor of $X \in \mathcal{M}$ for a fixed $Y \in \mathcal{M}$. It remains to check the commutativity of the diagram

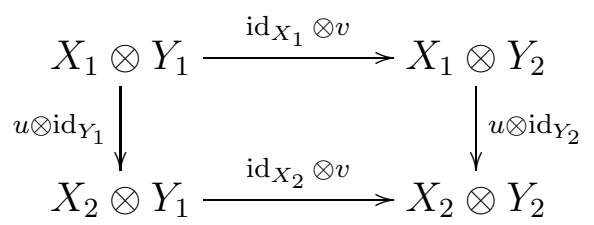

for all objects $X_{1}, X_{2}, Y_{1}, Y_{2} \in \mathcal{M}$ and morphisms $u: X_{1} \longrightarrow X_{2}$ and $v: Y_{1} \longrightarrow Y_{2}$ in $\mathcal{M}$. In fact, it suffices to do this when each of $u$ and $v$ is one of the "standard generators" of $\mathcal{M}$ (i.e., is either a morphism in $\mathcal{M}^{\prime}$, or $\delta: K^{\prime} \longrightarrow \mathbb{1}$, or $\pi: \mathbb{1} \longrightarrow \mathbb{1}^{\prime}$ ). The only nontrivial case is where $u=v=\delta$ (so that $X_{1}=Y_{1}=K^{\prime}$ and $X_{2}=Y_{2}=\mathbb{1}$ ). Here we are reduced precisely to the commutativity of (15.5).

The next result is obvious from the construction. 
Lemma 15.4. If the assumptions of Lemma 15.3 are satisfied and $\mathcal{M}$ is equipped with the monoidal category structure described in the lemma, then $\pi$ is an idempotent arrow in $\mathcal{M}$ and $\mathcal{M}^{\prime}$ is identified with the Hecke subcategory $\mathbb{1}^{\prime} \mathcal{M} \mathbb{1}^{\prime} \subset \mathcal{M}$.

Remark 15.5. The monoidal category $\mathcal{M}$ together with morphisms $K^{\prime} \stackrel{\delta}{\longrightarrow} \mathbb{1} \stackrel{\pi}{\longrightarrow} \mathbb{1}^{\prime}$, constructed above, can also be characterized by a universal property. Namely, suppose $\mathcal{N}$ is a monoidal category, $\varpi: \mathbb{1}_{\mathcal{N}} \longrightarrow e$ is an idempotent arrow, $F: \mathcal{M}^{\prime} \longrightarrow e \mathcal{N} e$ is a monoidal functor and $\xi: F\left(K^{\prime}\right) \longrightarrow \mathbb{1}_{\mathcal{N}}$ is a morphism such that the triangle

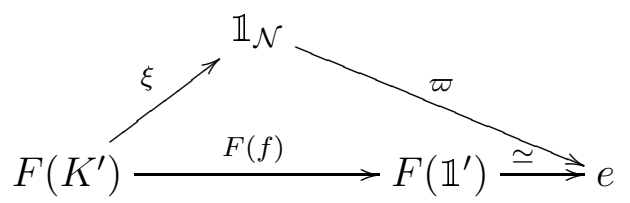

commutes. Then $F$ admits a unique extension to a monoidal functor $\mathcal{M} \longrightarrow \mathcal{N}$ such that $\delta \mapsto \xi$ and $\pi \mapsto \varpi$.

15.3. Proof of Proposition 9.4. In this subsection we assume that all the hypotheses of Proposition 9.4 are satisfied. Let $(\mathcal{M}, \mathbb{1}, \delta, \pi)$ be as in $\S 15.2$. The assumption that $D f=f$ is equivalent to the commutativity of the diagram (15.5), and we equip $\mathcal{M}$ with the monoidal structure described in Lemma 15.3. In view of Lemma 15.4, the proof of Proposition 9.4 will be complete once we establish

Lemma 15.6. Let $D$ denote the unique extension of the duality functor $D: \mathcal{M}^{\prime} \stackrel{\sim}{\longrightarrow} \mathcal{M}^{\prime}$ to a contravariant functor $D: \mathcal{M} \longrightarrow \mathcal{M}$ determined by $D(\mathbb{1})=\mathbb{1}, D(\delta)=\pi, D(\pi)=\delta$. Then $D: \mathcal{M} \longrightarrow \mathcal{M}$ is an anti-autoequivalence and there are functorial isomorphisms $\operatorname{Hom}(X \otimes Y, \mathbb{1}) \stackrel{\simeq}{\longrightarrow} \operatorname{Hom}(X, D Y)$ for all $X, Y \in \mathcal{M}$; in particular, $\mathcal{M}$ is an r-category.

Proof. The unique extension of $D^{-1}: \mathcal{M}^{\prime} \stackrel{\sim}{\longrightarrow} \mathcal{M}^{\prime}$ to a contravariant functor $\mathcal{M} \longrightarrow \mathcal{M}$ determined by $\mathbb{1} \mapsto \mathbb{1}, \delta \mapsto \pi, \pi \mapsto \delta$ is quasi-inverse to $D: \mathcal{M} \longrightarrow \mathcal{M}$; thus $D$ is an anti-autoequivalence of $\mathcal{M}$.

Next, consider the contravariant functors $F_{1}(X, Y)=\operatorname{Hom}(X \otimes Y, \mathbb{1})$ and $F_{2}(X, Y)=$ $\operatorname{Hom}(X, D Y)$ from $\mathcal{M} \times \mathcal{M}$ to the category of sets. By assumption, we have an isomorphism $\left.\left.F_{1}\right|_{\mathcal{M}^{\prime} \times \mathcal{M}^{\prime}} \stackrel{\simeq}{\longrightarrow} F_{2}\right|_{\mathcal{M}^{\prime} \times \mathcal{M}^{\prime}}$, since by construction, $\delta: K^{\prime} \longrightarrow \mathbb{1}$ identifies $\left.F_{1}\right|_{\mathcal{M}^{\prime} \times \mathcal{M}^{\prime}}$ with the functor $(X, Y) \mapsto \operatorname{Hom}\left(X \otimes Y, K^{\prime}\right)$. It is easy to check that this isomorphism extends to a unique isomorphism $F_{1} \stackrel{\simeq}{\longrightarrow} F_{2}$ such that when $Y=\mathbb{1}$, the induced map

$$
\operatorname{Hom}(X, \mathbb{1})=\operatorname{Hom}(X \otimes \mathbb{1}, \mathbb{1}) \stackrel{\simeq}{\longrightarrow} \operatorname{Hom}(X, D \mathbb{1})=\operatorname{Hom}(X, \mathbb{1})
$$

equals the identity, and when $X=\mathbb{1}$, the induced map

$$
\operatorname{Hom}(Y, \mathbb{1})=\operatorname{Hom}(\mathbb{1} \otimes Y, \mathbb{1}) \stackrel{\simeq}{\longrightarrow} \operatorname{Hom}(\mathbb{1}, D Y)
$$

equals $D: \operatorname{Hom}(Y, \mathbb{1}) \longrightarrow \operatorname{Hom}(D \mathbb{1}, D Y)=\operatorname{Hom}(\mathbb{1}, D Y)$. This proves the lemma. 
15.4. An example. We will show that in Proposition 9.4 the condition $D f=f$ does not hold automatically.

Let $k$ be a field, $X=\left\{(x, y) \in \mathbb{A}_{k}^{2} \mid x y=0\right\}$, and let $\mathcal{M}^{\prime}=\mathscr{D}(X)=D_{c}^{b}\left(X, \overline{\mathbb{Q}}_{\ell}\right)$ be the bounded derived category of constructible $\ell$-adic complexes on $X$ equipped with the usual (derived) tensor product $\otimes$. Put $K^{\prime}=K_{X}[-2]$, where $K_{X}$ is the dualizing complex of $X$. Then $\left(\mathcal{M}^{\prime}, K^{\prime}\right)$ is a Grothendieck-Verdier category, and we claim that there exists a morphism $f: K^{\prime} \longrightarrow \mathbb{1}^{\prime}$ such that $D f \neq f$.

Proof. Since $\mathcal{M}^{\prime}$, equipped with the standard symmetry isomorphisms $M \otimes N \stackrel{\simeq}{\longrightarrow} N \otimes M$, is a symmetric monoidal category, it follows that a morphism $f: K^{\prime} \longrightarrow \mathbb{1}^{\prime}$ satisfies $D f=$ $f$ if and only if the corresponding morphism $K^{\prime} \otimes K^{\prime} \longrightarrow K^{\prime}$ is symmetric. Thus we need to check that $\operatorname{Hom}\left(\bigwedge^{2} K^{\prime}, K^{\prime}\right) \neq 0$. We have $\operatorname{Hom}\left(\bigwedge^{2} K^{\prime}, K^{\prime}\right)=\operatorname{Hom}\left(\left(\bigwedge^{2} K_{X}\right)[-2], K_{X}\right)$. Since $\bigwedge^{2} K_{X}$ is concentrated at the singular point $0 \in X$, one has

$$
\operatorname{Hom}\left(\left(\bigwedge^{2} K_{X}\right)[-2], K_{X}\right)=\operatorname{Hom}\left(\bigwedge^{2}\left(K_{X}\right)_{0}[-2], \overline{\mathbb{Q}}_{\ell}\right),
$$

where $\left(K_{X}\right)_{0}$ is the stalk of $K_{X}$ at 0 . But $H^{-1}\left(\left(K_{X}\right)_{0}\right)=\overline{\mathbb{Q}}_{\ell}$ and $H^{i}\left(\left(K_{X}\right)_{0}\right)=0$ for $i \geq 0$, so $\operatorname{Hom}\left(\bigwedge^{2}\left(K_{X}\right)_{0}[-2], \overline{\mathbb{Q}}_{\ell}\right)=\operatorname{Hom}\left(\operatorname{Sym}^{2} \overline{\mathbb{Q}}_{\ell}, \overline{\mathbb{Q}}_{\ell}\right)=\overline{\mathbb{Q}}_{\ell}$.

\section{REFERENCES}

[BK01] B. Bakalov and A. Kirillov, Jr, "Lectures on tensor categories and modular functors", University Lecture Series 21. American Mathematical Society, Providence, RI, 2001.

[Ba79] M. Barr, *-autonomous categories (with an appendix by Po Hsiang Chu), Lect. Notes in Math. 752, Springer-Verlag, Berlin, 1979.

[Ba95] M. Barr, Nonsymmetric *-autonomous categories, Theoret. Comput. Sci. 139 (1995), no. 1-2, $115-130$.

[Ba96] M. Barr, *-autonomous categories, revisited, J. Pure Appl. Algebra 111 (1996), no. 1-3, 1-20.

[Ba99] M. Barr, *-autonomous categories: once more around the track, Theory Appl. Categ. 6 (1999), 5-24 (electronic).

[BD06] M. Boyarchenko and V. Drinfeld, A motivated introduction to character sheaves and the orbit method for unipotent groups in positive characteristic, Preprint, September 2006, arXiv: math.RT/0609769

[BD11] M. Boyarchenko and V. Drinfeld, Character sheaves on unipotent groups in positive characteristic: foundations, arXiv:0810.0794, version 2

[DS04] B. Day and R. Street, Quantum categories, star autonomy, and quantum groupoids, in: "Galois theory, Hopf algebras, and semiabelian categories", 187-225, Fields Inst. Commun. 43, Amer. Math. Soc., Providence, RI, 2004.

[De90] P. Deligne, Catégories tannakiennes, in: "The Grothendieck Festschrift", Vol. II, 111-195, Progr. Math. 87, Birkhäuser Boston, Boston, MA, 1990.

[EMC10] J. Egger and M.B. McCurdy, On Cyclic Star-Autonomous Categories, Preprint, July 2010, arXiv: 1007.5099

[Ek90] T. Ekedahl, On the adic formalism, in: "The Grothendieck Festschrift", vol. II, Progr. Math. 87, pp. 197-218, Birkhäuser, Boston, MA, 1990.

[ENO05] P. Etingof, D. Nikshych and V. Ostrik, On fusion categories, Annals of Math. 162 (2005), 581-642. 
[FY89] P. J. Freyd and D. N. Yetter, Braided compact closed categories with applications to lowdimensional topology, Adv. Math. 77 (1989), no. 2, 156-182.

[Jan88] U. Jannsen, Continuous étale cohomology, Math. Ann. 280 (1988), 207-245.

[JS93] A. Joyal and R. Street, Braided tensor categories, Adv. Math. 102 (1993), no. 1, 20-78.

[KS94] M. Kashiwara and P. Schapira, "Sheaves on manifolds", Grundlehren Math. Wiss. 292, SpringerVerlag, Berlin, 1994.

[LO06] Y. Laszlo and M. Olsson, The six operations for sheaves on Artin stacks II: Adic coefficients, Publ. Math. IHES. 107 (2008), 169-210.

[Lu09] J. Lurie, On the classification of topological field theories (version of May 10, 2009), available online at http://www.math.harvard.edu/ lurie/

[McL98] S. MacLane, "Categories for the working mathematician", 2nd ed. Graduate Texts in Mathematics 5. Springer-Verlag, New York, 1998. 